\title{
Energy-Spectral Efficiency Trade-Offs in Full-Duplex MU-MIMO Cloud-RANs with SWIPT
}

\author{
Xuan-Xinh Nguyen (iD) ${ }^{1,2}$ and Ha Hoang Kha ${ }^{1,2}$ \\ ${ }^{1}$ Ho Chi Minh City University of Technology (HCMUT), 268 Ly Thuong Kiet Street, District 10, Ho Chi Minh City, Vietnam \\ ${ }^{2}$ Vietnam National University Ho Chi Minh City, Linh Trung Ward, Thu Duc District, Ho Chi Minh City, Vietnam \\ Correspondence should be addressed to Xuan-Xinh Nguyen; nxxinh@hcmut.edu.vn
}

Received 19 October 2020; Revised 11 February 2021; Accepted 9 March 2021; Published 22 April 2021

Academic Editor: Felip Riera-Palou

Copyright (C) 2021 Xuan-Xinh Nguyen and Ha Hoang Kha. This is an open access article distributed under the Creative Commons Attribution License, which permits unrestricted use, distribution, and reproduction in any medium, provided the original work is properly cited.

\begin{abstract}
The present paper investigates the trade-offs between the energy efficiency (EE) and spectral efficiency (SE) in the full-duplex (FD) multiuser multi-input multioutput (MU-MIMO) cloud radio access networks (CRANs) with simultaneous wireless information and power transfer (SWIPT). In the considered network, the central unit (CU) intends to concurrently not only transfer both energy and information toward downlink (DL) users using power splitting structures but also receive signals from uplink (UL) users. This communication is executed via FD radio units (RUs) which are distributed nearby users and connected to the CU through limited capacity fronthaul (FH) links. In order to unveil interesting trade-offs between the EE and SE metrics, we first introduce three conventional single-objective optimization problems (SOOPs) including (i) system sum rate maximization, (ii) total power minimization, and (iii) fractional energy efficiency maximization. Then, by making use of the multiobjective optimization (MOO) framework, the MOO problem (MOOP) with the objective vector of the achievable rate and power consumption is addressed. All considered problems are nonconvex with respect to designing variables comprising precoding matrices, compression matrices, and DL power splitting factors; thus, it is extremely intractable to solve these problems directly. To overcome these issues, we develop iterative algorithms by utilizing the sequential convex approximation (SCA) approach for the first two SOO problems and the SCA-based Dinkelbach method for the fractional EE problem. Regarding the MOOP, we first rewrite it as an SOOP by applying the modified weighted Tchebycheff method and, then, propose the iterative algorithmbased SCA to find its optimal Pareto set. Various numerical simulations are conducted to study the system performance and appealing EE-SE trade-offs in the considered system.
\end{abstract}

\section{Introduction}

In the context of explosive growth in mobile data traffic and in wireless connectivities, the cloud radio access networks (CRANs) have been suggested as a modern architecture for the next generation of wireless communication systems. It is promising to overcome the strict requirements for concurrently enhancing the 1000 -fold data rate as well as reducing by half the power consumption [1-3]. Specifically, a typical CRAN composes of a central unit (CU), a set of distributed radio units (RUs), and terminal devices [1]. The $\mathrm{CU}$ is responsible for cooperatively processing baseband signals of all terminals and, then, communicates them to RUs through FH connections. These RUs which are inherently distributed nearby user equipment (UE) are responsible for transmitting or/and receiving data messages toward or/and from terminal devices. Based on this structure, the baseband signals are centrally processed at the $\mathrm{CU}$, and thus, CRANs provide not only a cooperative data transmission but also a better interference management among RUs as compared with the conventional ones, for example, multicell models $[4,5]$ and coordinated multipoint (CoMP) schemes [6, 7]. In addition, another remarkable advantage of CRANs is the replacement of the high-cost high-consuming power base stations by the lowcost low-consuming power RUs that can significantly reduce the system power consumption.

On the other hand, with potentially increasing the degrees of freedom in the design, the multiple antenna transmission 
techniques can thus support to convey more data streams over a same radio resource and promisingly boost the system throughput. Hence, the multiantenna techniques have been integrated and investigated in the context of CRANs. The CRANs implemented with multiple-antenna RUs and singleantenna terminals are named as MISO CRANs and investigated in [8-13]. Meanwhile, the ones deployed with multipleantenna RUs and multiple-antenna UEs were referred to as MIMO CRANs and studied in [14-17]. Regarding the MISO CRAN deployment, [8] proposed the joint design of RU activation and beamformers to minimize power consumption in downlink (DL) MISO CRAN systems. In [9], the authors first proposed a spatial-compression-and-forward strategy for uplink (UL) MISO CRAN systems and, then, addressed the beamforming and compressing design to maximize the minimum user's quality of service (QoS). Alternatively, the work in [10] developed a nonorthogonal multiple access (NOMA) transmission in DL MISO CRANs and, then, proposed a solution to handle the maximin individual user rate subjected to transmit power budget and limited FH capacity constraints. In addition, a wireless-powered MISO CRAN system was developed in [11] where the terminal devices conduct both information decoding (ID) and energy harvesting (EH) in the first ID-EH DL phase and each user then transmits its own data in the UL data transmission phase using harvested energy. The UL rate maximization problem is tackled under minimum DL rate requirements, power budget, and $\mathrm{FH}$ capacity constraints. Several novel techniques including hybrid digital-analog beamforming and rate splitting multiple access were addressed in DL MISO CRANs in $[12,13]$, respectively. As the result, the system spectral efficiency (SE) is thus considerably improved. On the other hand, by considering the MIMO CRAN scheme, the joint design of precoding and compressing matrices was investigated for the UL MIMO CRAN in $[14,15]$ and for the DL MIMO CRAN in [16], respectively. Additionally, the authors in [17] took multicluster interference into consideration and developed an effective algorithm to handle them in the multicluster DL MIMO CRAN.

It is worth noting that the aforementioned works separately studied the UL CRAN channels or DL CRAN ones and can be referred to as half-duplex (HD) transmission that leads to inefficient spectrum usage. Recently, thanks to the advanced analog and digital self-interference (SI) cancellation strategies, the full-duplex (FD) radio is able to concurrently transmit and receive signals over the same radio resources. Then, the FD techniques potentially provides the SE approximately twice as much as that of the conventional HD counterparts $[18,19]$. The integration between the FD radio approach and CRAN scheme has recently drawn significant attention [20-26]. Reference [20] investigated a classical Wyner model based on FD-aided CRANs and illustrated that the achievable rate of the FD CRAN system is significantly improved in comparison to both HD CRANs and single-cell processing ones. Reference [21] developed a two-stage algorithm to jointly design RU beamformers, user power allocation, and user selection for the FD MISO CRAN. Furthermore, the system data rate for the multiuser scheme was analysed and optimal power allocation for the single-user case was investigated in the FD CRANs [22]. Additionally, by considering a hybrid
RU set which comprises both multiantenna and singleantenna RUs, [23] aimed for joint resource allocation and interference cancellation in order to maximize the DL rate subjected to power and UL rate QoS constraints in the FD CRANs. By exploiting the difference of the convex (DC) form in the system data rate and $\mathrm{FH}$ capacities, the authors in [24] proposed an iterative algorithm based on the majorizationminimization method for the FD MIMO CRANs which jointly optimizes precoding and compressing matrices under transmit power and FH rate constraints. Alternatively, the work in [25] developed a low-complexity design for FD MIMO CRANs by making the use of the connection between weighted minimum mean squared error (WMMSE) problem and weighted sum rate (WSR) problem. The precoders and compressing matrices are then alternatively optimized by solving the convex problem in each iteration. Most recently, in [26], the simultaneous wireless information and power transfer (SWIPT) protocol was investigated on the FD MISO CRANs. The joint design of DL beamforming, EH-power splitting ratio, UL power allocation, and RU postcoding was addressed. Reference [27] investigated the energy-throughput trade-off by designing the beamforming for SWIPT CRANs. Alternatively, the authors in [28] developed the hybrid time-power splitting protocol for downlink user information and uplink ID-EH sensor in FD-aided massive MIMO wireless systems.

However, the aforementioned works were concentrated only on designing either the sum rate maximization (referred to as SE maximization) or the power consumption minimization in the considered CRAN. This might lead to ineffective and negative impacts on the system EE performance [29]. The EE issue was, therefore, taken into account in the transceiver design for CRAN networks [30-36]. Specifically, reference [30] investigated the resource allocation in order to minimize the entire system power consumption for both data sharing- and data compressing-based DL CRAN models. The authors in [31] first generalized these models to the multihop FH links MIMO CRANs and, then, proposed a successive convex quadratic programming (SCQP) algorithm to jointly design precoders, user-RU association, and RU activation to optimize the system fractional EE defined as a ratio of the system sum rate to power consumption. In order to further reduce power consumed by ineffective RUs, the issues of turning RU on/off and user-RU association were studied in various recent works. Particularly the joint design of precoding matrices, turning RUs on/off was investigated for both SE and EE optimization problems in DL MIMO CRAN-based multihop FH link networks [32]. In [33], the EE maximization problem constrained with $\mathrm{FH}$ capacity limitation, transmit power budget, and user's QoS requirements was tackled for a DL MISO CRAN. Alternatively, the EE beamforming design-based RU selecting and RU-UE associating designs for DL MISO CRAN were proposed either with virtual computing resource models in [34] or with $\mathrm{FH}$ rate-dependent power consumption models in [35]. To take advantage of FD techniques, reference [36] proposed an efficient algorithm for optimizing the system EE in FD MISO CRANbased data sharing systems. However, the FH capacity constraints and $\mathrm{FH}$ power consumption model were not taken into consideration. 
We would like to emphasize that, in the aforementioned works, only one specific system performance metric is considered as a single-objective function in design problems while the others are taken into account in the constraints. This approach can be referred to as single-objective optimization (SOO) design and can lead to an unfairness between the conflicting system measures, such as systems SE, EE and power consumption. More recently, the multiobjective optimization (MOO) framework has been introduced as an effective mathematical tool to overcome this issue as well as to provide a balance among the system performance metrics on designing the wireless communication systems $[37,38]$. As a result, the MOO framework was recently exploited in many studies to address various trade-offs between two or more system metrics [39-48]. Particularly, the harvested energy and information transfer trade-offs in SWIPT-enabled wireless system were studied either in cognitive FD MU-MIMO systems [39] or in MIMO broadcast channels [40, 41]. In [42], the authors studied the optimal Pareto set for DL and UL transmit power fairness in the FD MISO networks. Furthermore, the balance between transmit power and achievable rate was investigated in $[43,44]$ for secure DL MU-MISO networks and DL MISO CRANs, respectively. In addition, the EE-SE trade-offs for massive MIMO systems were addressed in $[45,46]$. On the other hand, the works in $[47,48]$ have developed algorithms using MOO frameworks to study the balance among three essential and conflicting metrics. Particularly, the balance among three inconsistent design objectives of the MSE minimization, harvested energy maximization, and physical security optimization was revealed for SWIPT-aided MIMO interference channels in [47]. Furthermore, the work in [48] addressed the fairness among total transmit power minimization, EH efficiency maximization, and interference leakage-totransmit power ratio minimization for SWIPT-based MISO cognitive radio networks.

Motivated by the above viewpoints, this paper studies EE-SE trade-offs in an FD MU-MIMO CRAN-enabling SWIPT network (shown in Figure 1). The considered CRAN model includes that a set of FD RU stations, which is connected to the $\mathrm{CU}$ through the limited capacity FH links, concurrently communicates to the sets of EH-enabled DL users (EH-DLUs) and UL users (ULUs). Specifically, regarding DL channels, the baseband signals associating with DLUs are first centrally processed at the $\mathrm{CU}$ and, then, conveyed to the RUs via FH links. After radio frequency (RF) steps, the distributed RUs transmit these signals toward DLUs through wireless access links. At the DLU, the received signal is divided into two proportions using power splitting (PS) receiver structures, which are then performed for ID and $\mathrm{EH}$ purposes. The total amount of harvested energy can be stored at the DLUs to prolong their lifetime or to utilize for signal processing operations as discussed in previous works $[26,39,47,48]$. In the UL channels, the individual-received baseband signals at RUs are sent to the $\mathrm{CU}$ via FH links for central processing. It is noted that, in order to guarantee the rate over finite capacity FH links, the baseband signals are quantized and compressed by using $\mathrm{FH}$ compression strategies [14-16]. Hence, we aim to jointly design precoding matrices, FH compressing covariance matrices, and power

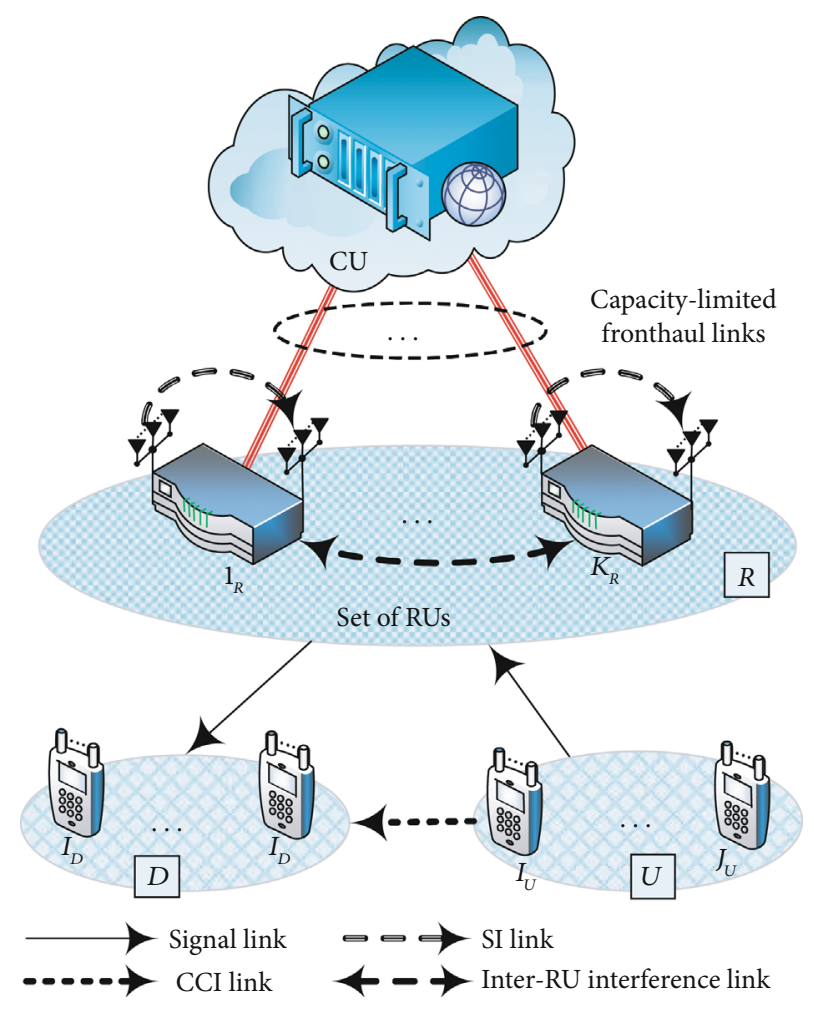

Figure 1: An illustration of the SWIPT-enabled FD MU-MIMO CRAN system.

splitting parameters. Moreover, to investigate the trade-offs between two conflicting EE and SE metrics, we introduce three SOO problems (SOOPs) and one MOO problem (MOOP); they are all under constraints of transmit power budget, minimum data rate, maximum FH link capacity, and minimum amount of harvested energy. Specifically, the SOOPs include (i) sum rate maximization, (ii) power consumption minimization, and (iii) fractional energy efficiency maximization whereas the MOOP is the simultaneous sum rate and power consumption optimization one. All interesting problems are the challenging nonconvex optimization ones and hard to be directly handled. This is due not only to the nonconcavity in data rate functions and nonconvexity in FH link rates caused by the coupled of UL and DL precoder variables but also to the inverse convexity in $\mathrm{EH}$ constraints. To overcome these issues, we exploit the efficient inner convex approximation and SCQP approaches to develop iterative algorithms with low computational complexity.

The main contributions of this work are summarized as twofold as follows:

(1) This paper attempts to comprehensively study the SE-EE trade-off in the FD MIMO CRANs with SWIPT. Compared to previous related works in the literatures $[24-26,36]$, we highlight the novelty of the present work as follows:

(i) Although the works in $[24,25]$ have addressed the transceiver design for FD CRANs in SE perspectives, the present paper first extends the 
system model for enabling SWIPT at DLUs and, then, studies the additional system EE metric using two approaches, namely, the maximization of the ratio between the sum rate over the power consumption based on the SOO programming and simultaneous sum rate maximization and power consumption minimization based on MOO programming.

(ii) On the other hand, it is worth noting that the work [26] has also attempted to minimize power beamforming under the QoS of the data rate and harvested energy in SWIPT-enabled FD MISO CRANs while the authors in [36] have also proposed an algorithm to optimize the overall system EE in FD MISO CRANs. However, both works assumed single-antenna users and unlimitedcapacity FH links, therefore, those approaches cannot be directly applied for FD MIMO CRANs with SWIPT considered in this paper.

Thus, the present work is distinguished itself and can be referred to as the more comprehensive investigation

(2) We also develop a computationally efficient iterative algorithm based on the SCQP approach, in which the log-determinant expressions in both objective function and $\mathrm{FH}$ rate constraints are recast by the quadratic convex ones within each iteration. As the result, the computational cost, which is essentially caused by log-determinant expressions, is significantly reduced as compared with the methods in $[24,25]$.

The rest of this paper is organized as follows. Section 2 introduces the system model and transmission signaling and presents the problem formulations for SOOPs, which include sum rate maximization, power consumption minimization, and energy efficiency maximization, as well as introduces an EE-SE trade-off MOOP. Section 3 develops algorithms for considered SOOPs and MOOP. Finally, the simulation results and conclusions are provided in Section 4 and Section 5, respectively.

Notations. Bold lower- and uppercased letters represent vectors and matrices, respectively. $\mathbf{Y}^{T}, \mathbf{Y}^{\dagger}, \operatorname{Tr}(\mathbf{Y})$, and $|\mathbf{Y}|$ are the transpose, Hermitian transpose, trace, and determinant of $\mathbf{Y}$, respectively. The notation $\mathbf{Y} \succeq \mathbf{M}(\mathbf{Y}>\mathbf{M}$, respectively) means that $\mathbf{Y}-\mathbf{M}$ is a positive semidefinite (positive definite, respectively) matrix. $\mathbf{I}_{n}$ represents the $n \times n$ identical matrix. We denote $\mathbf{M}^{(\kappa),-1} \triangleq\left(\mathbf{M}^{(\kappa)}\right)^{-1}$ and $\mathbf{M}^{(\kappa), \dagger} \triangleq\left(\mathbf{M}^{(\kappa)}\right)^{\dagger}$. Operator $\mathfrak{R}\{x\}$ is the real part of the complex number $x$ and $\mathbb{E}\{\cdot\}$ denotes the expectation operator.

\section{System Model}

This paper considers an FD MIMO CRAN-enabled SWIPT as shown in Figure 1, in which two sets of UL and DL users communicate with a CU through a pool of RUs. It is assumed that the limited-capacity FH links are used in CU-RU con- nections. Hence, to meet the bottleneck rate constraint, the data is compressed before being transferred over FH links. For notation simplicity, the sets of $K$ RUs, $I$ DLUs, and $J$ ULUs are denoted as $\mathscr{R} \triangleq\left\{\lambda_{R}: \lambda=1,2, \cdots, K\right\}, \mathscr{D} \triangleq\left\{k_{D}: k\right.$ $=1,2, \cdots, I\}$, and $\mathcal{U} \triangleq\left\{\ell_{U}: \ell=1,2, \cdots, J\right\}$, respectively. The number of transmit and receive antennas at RU $\lambda_{R}$ are $N_{\lambda_{R}}^{T x}$ and $N_{\lambda_{R}}^{R x}$, respectively. Thus, the numbers of all transmit and all receive antennas at RUs are $N_{R}^{T x}=\sum_{\lambda_{R} \in \mathscr{R}} N_{\lambda_{R}}^{T x}$ and $N_{R}^{R x}=\sum_{\lambda_{R} \in \mathscr{R}} N_{\lambda_{R}}^{R x}$, respectively. In addition, the numbers of transmit antennas of ULU $\ell_{U}$ and receive antennas of DLU $k_{D}$ are denoted as $N_{\ell_{U}}^{T x}$ and $N_{k_{D}}^{R x}$, respectively. Moreover, the channel state information (CSI) of all links is assumed to be fully known at the CU by exploiting the joint UL and DL channel estimation algorithm [49]. It should be noticed that such an assumption of the perfect CSI is widely adopted in the recent related works [9-17, 23-26]. In practice, the CSI can be achieved through the channel estimation, feedback, or reverse links, and thus, the imperfect CSI is available at the CU. In such cases, the results in this work can be referred to as the upper-bound performances.

At the same time and over the same frequency resource, the $\mathrm{CU}$ intends to transmit the message $\mathbf{s}_{k_{D}} \in \mathbb{C}^{d_{k_{D}} \times 1}$ for DLU $k_{D}$ in the DL channels while the ULU also wishes to convey its own message $s_{\ell_{U}} \in \mathbb{C}^{d_{\ell_{U}} \times 1}$ to the $\mathrm{CU}$, with $d_{k_{D}}$ and $d_{\ell_{U}}$ representing the numbers of data streams of DLU $k_{D}$ and ULU $\ell_{U}$, respectively. All data symbols are assumed with zero mean and unit power-independent distribution, i.e., $\mathbb{E}\left\{\mathbf{s}_{i} \mathbf{s}_{j}^{\dagger}\right\}=\mathbf{0}$ with $i \neq j$ and $\mathbb{E}\left\{\mathbf{s}_{i} \mathbf{s}_{i}^{\dagger}\right\}=\mathbf{I}$ with an appropriate dimension for all $i, j \in\{\mathscr{U}, \mathscr{D}\}$. In addition, to take advantages of multiantenna transmission in spatial diversity enhancement, the DL and UL signals are first beamformed by multiplying with its corresponding precoding matrices, namely, $\mathbf{F}_{k_{D}} \in \mathbb{C}^{N_{R}^{T x} \times d_{k_{D}}}$ for DLU $k_{D}$ and $\mathbf{F}_{\ell_{U}} \in \mathbb{C}^{N_{\ell_{U}}^{T x} \times d_{\ell_{U}}}$ for ULU $\ell_{U}$. Thus, in the UL channels, the transmit signal at ULU $\ell_{U}$ is given as $\mathbf{x}_{\ell_{U}}=\mathbf{F}_{\ell_{U}} s_{\ell_{U}}$. Then, the power constraints at ULUs, i.e., $\mathbb{E}\left\|\mathbf{x}_{\ell_{U}}\right\|^{2} \leq P_{\ell_{U}}, \forall \ell_{U} \in \mathscr{U}$, are determined by

$$
P_{\ell_{U}}^{T x} \triangleq\left\|\mathbf{F}_{\ell_{U}}\right\|^{2} \leq P_{\ell_{U}}, \quad \forall \ell_{U} \in \mathscr{U}
$$

Concerning the DL channels, it is emphasized that the precoding matrix for DLU $k_{D}$ is constructed at the $\mathrm{CU}$ in the form $\mathbf{F}_{k_{D}}^{\dagger} \triangleq\left[\mathbf{F}_{k_{D}, 1_{R}}^{\dagger}, \cdots, \mathbf{F}_{k_{D}, K_{R}}^{\dagger}\right][16,17]$, in which $\mathbf{F}_{k_{D}, \lambda_{R}} \in$ $\mathbb{C}^{N_{\lambda_{R}}^{T x} \times d_{k_{D}}}$ is the precoder matrix for DLU $k_{D}$ at RU $\lambda_{R}$. Hence, the stacked DL data vector at RUs is given by $\tilde{\mathbf{x}}_{D}=\sum_{k_{D} \in \mathscr{D}}$ $\mathbf{F}_{k_{D}} \mathbf{s}_{k_{D}}=\left[\tilde{\mathbf{x}}_{D, 1_{R}}^{\dagger}, \tilde{\mathbf{x}}_{D, 2_{R}}^{\dagger}, \cdots, \tilde{\mathbf{x}}_{D, K_{R}}^{\dagger}\right]^{\dagger}$ and the DL data vector corresponding to RU $\lambda_{R}$ is given as $\tilde{\mathbf{x}}_{D, \lambda_{R}} \triangleq \mathbf{E}_{\lambda_{R}}^{\dagger} \sum_{k_{D} \in \mathscr{D}} \mathbf{F}_{k_{D}} \mathbf{s}_{k_{D}}$ with

$$
\mathbf{E}_{\lambda_{R}}^{\dagger}=\left[\mathbf{0}_{\sum_{\theta-1}^{\dagger} N_{\theta_{R}}^{T x} \times N_{\lambda_{R}}^{T x}, \mathbf{I}_{N_{\lambda_{R}}^{T x}}^{\dagger}, \mathbf{0}_{K_{R}}^{\dagger} N_{\theta_{R}}^{T x} \times N_{\lambda_{R}}^{T x}}\right] .
$$


Prior to being conveyed throughout the limited capacity FH link, the DL signal for RU $\lambda_{R}$ is quantized and compressed. Adopting a Gaussian test channel, the compressed DL signal for $\mathrm{RU} \lambda_{R}$ is given as

$$
\mathbf{x}_{D, \lambda_{R}}=\tilde{\mathbf{x}}_{D, \lambda_{R}}+\mathbf{q}_{D, \lambda_{R}}=\mathbf{E}_{\lambda_{R}}^{\dagger} \sum_{k_{D} \in \mathscr{D}} \mathbf{F}_{k_{D}} \mathbf{s}_{k_{D}}+\mathbf{q}_{D, \lambda_{R}}, \quad \forall \lambda_{R} \in \mathscr{R},
$$

where the quantization noise of DL FH link $\lambda_{R}, \mathbf{q}_{D, \lambda_{R}}$, is independent of precoded data stream and obeys $\mathbf{q}_{D, \lambda_{R}} \sim \mathscr{C}$ $\mathcal{N}\left(\mathbf{0}, \mathbf{Q}_{D, \lambda_{R}}\right)$ as in $[16,17,24,25]$. This process is to guarantee the rate constraint over the FH link. Hence, the power constraint at RUs, i.e., $\mathbb{E}\left\|\mathbf{x}_{D, \lambda_{R}}\right\|^{2} \leq P_{\lambda_{R}}$ with $\lambda_{R} \in \mathscr{R}$, is equivalently expressed as

$$
P_{\lambda_{R}}^{T x} \triangleq \sum_{k_{D} \in \mathscr{D}}\left\|\mathbf{E}_{\lambda_{R}}^{\dagger} \mathbf{F}_{k_{D}}\right\|^{2}+\operatorname{Tr}\left(\mathbf{Q}_{D, \lambda_{R}}\right) \leq P_{\lambda_{R}}, \quad \forall \lambda_{R} \in \mathscr{R} .
$$

2.1. DL Channels with Information and Energy Transferring. From (3), the noiseless FH rate of the link from CU to RU $\lambda_{R}$ is determined as $[16,17]$

$$
g_{D, \lambda_{R}}=\log _{2}\left|\mathbf{I}_{N_{\lambda_{R}}^{T x}}+\mathbf{Q}_{D, \lambda_{R}}^{-1} \sum_{k_{D} \in \mathscr{D}} \mathbf{E}_{\lambda_{R}}^{\dagger} \mathbf{F}_{k_{D}} \mathbf{F}_{k_{D}}^{\dagger} \mathbf{E}_{\lambda_{R}}\right|
$$

In addition, the received signal vector at DLU $k_{D}, \mathbf{y}_{k_{D}} \epsilon$ $\mathbb{C}^{N_{k_{D}}^{R x} \times 1}$, is given as

$$
\mathbf{y}_{k_{D}}=\mathbf{H}_{k_{D}} \mathbf{x}_{D}+\sum_{\ell_{U} \in \mathcal{U}} \mathbf{G}_{k_{D}, \ell_{U}} \mathbf{x}_{\ell_{U}}+\mathbf{n}_{k_{D}, a},
$$

where $\mathbf{x}_{D}^{\dagger}=\left[\mathbf{x}_{D, 1_{R}}^{\dagger}, \mathbf{x}_{D, 2_{R}}^{\dagger}, \cdots, \mathbf{x}_{D, K_{R}}^{\dagger}\right]$ and $\mathbf{H}_{k_{D}}=\left[\mathbf{H}_{k_{D}, 1_{R}}, \mathbf{H}_{k_{D}, 2_{R}}\right.$, $\left.\cdots, \mathbf{H}_{k_{D}, K_{R}}\right] \in \mathbb{C}^{N_{k_{D}}^{R x} \times N_{R}^{T x}}$ denote the channel matrix from RUs to DLU $k_{D}$ with $\mathbf{H}_{k_{D}, \lambda_{R}} \in \mathbb{C}^{N_{k_{D}}^{R x} \times N_{\lambda_{R}}^{T x}}$ being the complex channel matrix between RU $\lambda_{R}$ and DLU $k_{D}$ while $\mathbf{G}_{k_{D}, \ell_{U}} \in$ $\mathbb{C}^{N_{k_{D}}^{R x} \times N_{\ell_{U}}^{T x}}$ represents the CCI channel coefficient to DLU $k_{D}$ from ULU $\ell_{U}$ and $\mathbf{n}_{k_{D}, a} \in \mathbb{C}^{N_{k_{D}}^{R x} \times 1}$ represents the additive Gaussian noise vector at DLU $k_{D}$ with zero mean and covariance matrix $\sigma_{k_{D}, a}^{2} \mathbf{I}_{N_{k_{D}}^{R x}}$.In order to realize SWIPT via received RF signals, this paper adopts power splitting receiver structures at DLUs. Specially, we exploit perantenna power splitting (PAPS) structures in EH-DLUs. Denoting $0 \leq w_{k_{D}, j} \leq 1$ as the signal proportion factor for ID at antenna $j$ of DLU $k_{D}$ with $j=1,2, \cdots, N_{k_{D}}^{R x}$, the PAPS coefficients of DLU $k_{D}$ are represented by a diagonal matrix $\mathbf{W}_{I, k_{D}}=\operatorname{diag}\left\{w_{k_{D}, 1}, w_{k_{D}, 2}, \cdots, w_{k_{D}, N_{k_{D}}^{R x}}\right\}$ which satisfies $\mathbf{W}_{I, k_{D}}$ $\geq \mathbf{0}_{N_{k_{D}}^{R x}}$ and $\mathbf{I}_{N_{k_{D}}^{R x}} \geq \mathbf{W}_{I, k_{D}}$. Then, the received signal for ID is

$$
\mathbf{y}_{k_{D}}^{I D}=\mathbf{W}_{k_{D}}^{0.5} \mathbf{y}_{k_{D}}+\mathbf{n}_{k_{D}, c},
$$

where $\mathbf{n}_{k_{D}, c} \in \mathbb{C}^{N_{k_{D}}^{R x} \times 1}$ represents the additive circuitry Gaussian noise vector at DLU $k_{D}$ with zero mean and covariance matrix $\sigma_{k_{D}, c}^{2} \mathbf{I}_{N_{k_{D}}^{R x}}$. Accordingly, the residual amount of signals is used for scavenging energy. Therefore, the signal for the $\mathrm{EH}$ purpose is given as

$$
\mathbf{y}_{k_{D}}^{\mathrm{EH}}=\left(\mathbf{I}_{N_{k_{D}}^{R x}}-\mathbf{W}_{k_{D}}\right)^{0.5} \mathbf{y}_{k_{D}} \text {. }
$$

From (7), the DL rate at DLU $k_{D}$ can be expressed as the function of precoders, compression matrices, and PAPS matrices and given by

$$
R_{k_{D}}=\log _{2}\left|\mathbf{I}_{N_{k_{D}}^{R x}}+\left(\boldsymbol{\Omega}_{k_{D}}+\sigma_{k_{D}, c}^{2} \mathbf{W}_{k_{D}}^{-1}\right)^{-1} \mathbf{C}_{k_{D}}\right|,
$$

where $\mathbf{C}_{k_{D}} \triangleq \mathbf{H}_{k_{D}} \mathbf{F}_{k_{D}} \mathbf{F}_{k_{D}}^{\dagger} \mathbf{H}_{k_{D}}^{\dagger}$ denotes the covariance matrix of the desired downlink signal and

$$
\begin{aligned}
\boldsymbol{\Omega}_{k_{D}} \triangleq & \sum_{i_{D} \in \mathscr{D} /\left\{k_{D}\right\}} \mathbf{H}_{k_{D}} \mathbf{F}_{i_{D}} \mathbf{F}_{i_{D}}^{\dagger} \mathbf{H}_{k_{D}}^{\dagger}+\mathbf{H}_{k_{D}} \mathbf{Q}_{D} \mathbf{H}_{k_{D}}^{\dagger} \\
& +\sum_{\ell_{U} \in \mathscr{U}} \mathbf{G}_{k_{D}, \ell_{U}} \mathbf{F}_{\ell_{U}} \mathbf{F}_{\ell_{U}}^{\dagger} \mathbf{G}_{k_{D}, \ell_{U}}^{\dagger}+\sigma_{k_{D}, a}^{2} \mathbf{I}_{N_{k_{D}}^{R x}},
\end{aligned}
$$

which is the covariance matrix of multiuser, cochannel, and compression interferences plus additive noise at DLU $k_{D}$, where we have defined $\mathbf{Q}_{D} \triangleq \operatorname{diag}\left\{\mathbf{Q}_{D, \lambda_{R}}, \lambda_{R} \in \mathscr{R}\right\}$.Moreover, upon (8), the amount of harvested energy at DLU $k_{D}$ is expressed as follows

$$
E_{k_{D}}=\operatorname{Tr}\left(\left(\mathbf{I}_{N_{k_{D}}^{R x}}-\mathbf{W}_{k_{D}}\right) \boldsymbol{\Pi}_{k_{D}}\right)
$$

where the DL signal covariance mapping matrix $\Pi_{k_{D}}=\mathbf{C}_{k_{D}}$ $+\boldsymbol{\Omega}_{k_{D}}$. In this paper, we consider a practical nonlinear model of energy harvester which is mathematically formulated as [50]

$$
E_{k_{D}}^{\mathrm{NL}}=\frac{\Delta_{k_{D}}}{1+\exp \left(-a_{k_{D}} E_{k_{D}}+a_{k_{D}} b_{k_{D}}\right)}-\Delta_{k_{D}} \Omega_{k_{D}}
$$

where $\Delta_{k_{D}}=\bar{P}_{k_{B}}^{D C}\left(1-\Omega_{k_{D}}\right)^{-1}$ with $\Omega_{k_{D}}=\left(1+\exp \left(a_{k_{D}} b_{k_{D}}\right)\right)^{-1}$ . Meanwhile, $\bar{P}_{k_{D}}^{D C}$ denotes the maximum power harvested at DLU $k_{D}$ at the saturation point of EH circuit and $a_{k_{D}}$ and $b_{k_{D}}$ are the nonlinear $\mathrm{EH}$ parameters by hardware limitations as illustrated in [50]. This quantity of energy, $E_{k_{D}}^{\mathrm{NL}}$, can be stored either in a battery or in a super capacitor and later exploited to prolong its own operation such as processing/decoding signals or transmitting control signals to the RUs $[26,39,47,48,50,51]$.

2.2. UL Channels with Information Transferring. As the transmission strategy discussed in the early subsection, the 
received baseband signal at RU $\lambda_{R}$ in UL channels is given as

$$
\mathbf{y}_{\lambda_{R}}=\sum_{\ell_{U} \in \mathscr{U}} \mathbf{H}_{\lambda_{R}, \ell_{U}} \mathbf{x}_{\ell_{U}}+\mathbf{G}_{\lambda_{R}, R} \mathbf{x}_{D}+\mathbf{n}_{\lambda_{R}}
$$

where $\mathbf{H}_{\lambda_{R}, \ell_{U}} \in \mathbb{C}^{N_{\lambda_{R}}^{R x} \times N_{\ell_{U}}^{T x}}$ is the channel gain between ULU $\ell_{U}$ and RU $\lambda_{R}$ whereas $\mathbf{G}_{\lambda_{R}, R} \triangleq\left[\mathbf{G}_{\lambda_{R}, 1_{R}}, \mathbf{G}_{\lambda_{R}, 2_{R}}, \cdots, \mathbf{G}_{\lambda_{R}, K_{R}}\right] \epsilon$ $\mathbb{C}^{N_{\lambda_{R}}^{T x} \times N_{R}^{R x}}$ with $\mathbf{G}_{\lambda_{R}, \theta_{R}} \in \mathbb{C}^{N_{\lambda_{R}}^{R x} \times N_{\theta_{R}}^{T x}}$ is the interference link from RU $\theta_{R}$ transmission to RU $\lambda_{R}$ reception. Specifically, if $\theta_{R}$ $\equiv \lambda_{R}$, interference is referred to as SI at RU $\lambda_{R}$; otherwise, it is an inter-RU interference link between receive antennas at RU $\lambda_{R}$ and transmit antennas at RU $\theta_{R}$. The additive Gaussian noise at RU $\lambda_{R}$ is denoted as $\mathbf{n}_{\lambda_{R}} \in \mathbb{C}^{N_{\lambda_{R}}^{R x} \times 1}$ with zero mean and covariance matrix $\sigma_{\lambda_{R}}^{2} \mathbf{I}_{N_{\lambda_{R}}^{T x}}$. Then, by adopting a Gaussian test channel compression model, the compressed version of the UL signal for the FH link $\lambda_{R}$ is $\tilde{\mathbf{y}}_{\lambda_{R}}=\mathbf{y}_{\lambda_{R}}+\mathbf{q}_{U, \lambda_{R}}$ where $\mathbf{q}_{U, \lambda_{R}} \sim \mathscr{C} \mathscr{N}\left(\mathbf{0}, \mathbf{Q}_{U, \lambda_{R}}\right)$ is the quantization noise which is randomized and independent from the received data stream [14, $15,24,25]$. The rate corresponding to UL FH link $\lambda_{R}$ is thus expressed as

$$
g_{U, \lambda_{R}}=\log _{2}\left|\mathbf{I}_{N_{\lambda_{R}}^{R x}}+\mathbf{Q}_{U, \lambda_{R}}^{-1} \boldsymbol{\Xi}_{U, \lambda_{R}}\right|
$$

where

$$
\begin{aligned}
\boldsymbol{\Xi}_{U, \lambda_{R}}= & \sum_{\ell_{U} \in \mathscr{U}} \mathbf{H}_{\lambda_{R}, \ell_{U}} \mathbf{F}_{\ell_{U}} \mathbf{F}_{\ell_{U}}^{\dagger} \mathbf{H}_{\lambda_{R}, \ell_{U}}^{\dagger}+\sum_{k_{D} \in \mathscr{D}} \mathbf{G}_{\lambda_{R}, R} \mathbf{F}_{k_{D}} \mathbf{F}_{k_{D}}^{\dagger} \mathbf{G}_{\lambda_{R}, R}^{\dagger} \\
& +\sum_{\theta_{R} \in \mathscr{R}} \mathbf{G}_{\lambda_{R}, \theta_{R}} \mathbf{Q}_{D, \theta_{R}} \mathbf{G}_{\lambda_{R}, \theta_{R}}^{\dagger}+\sigma_{\lambda_{R}}^{2} \mathbf{I}_{N_{\lambda_{R}}^{R x}}
\end{aligned}
$$

Define $\mathbf{H}_{R, \ell_{U}} \triangleq\left[\mathbf{H}_{1_{R}, \ell_{U}}^{\dagger}, \mathbf{H}_{2_{R}, \ell_{U}}^{\dagger}, \cdots, \mathbf{H}_{K_{R}, \ell_{U}}^{\dagger}\right]^{\dagger} \in \mathbb{C}^{N_{R}^{R x} \times N_{\ell_{U}}^{T x}}$ as the channel link from ULU $\ell_{U}$ to RUs. $\mathbf{G}_{R, R} \triangleq$ $\left[\mathbf{G}_{1_{R}, R}^{\dagger}, \mathbf{G}_{2_{R}, R}^{\dagger}, \cdots, \mathbf{G}_{K_{R}, R}^{\dagger}\right]^{\dagger} \in \mathbb{C}^{N_{R}^{R x} \times N_{R}^{T x}}$ represents the aggregative link from transmit to receive antennas between RUs. In addition, $\mathbf{q}_{U}^{\dagger} \triangleq\left[\mathbf{q}_{U, 1_{R}}^{\dagger}, \mathbf{q}_{U, 2_{R}}^{\dagger}, \cdots, \mathbf{q}_{U, K_{R}}^{\dagger}\right], \mathbf{q}_{D}^{\dagger} \triangleq\left[\mathbf{q}_{D, 1_{R}}^{\dagger}, \mathbf{q}_{D, 2_{R}}^{\dagger}, \cdots, \mathbf{q}_{D, K_{R}}^{\dagger}\right]$ , and $\mathbf{n}_{R}^{\dagger} \triangleq\left[\mathbf{n}_{1_{R}}^{\dagger}, \mathbf{n}_{1_{R}}^{\dagger}, \cdots, \mathbf{n}_{K_{R}}^{\dagger}\right]$ are the stacked versions of UL, DL quantization noise, and additive noise at RUs. Then, the stacked UL baseband signal vector received at all RUs, i.e., $\mathbf{y}_{R} \triangleq\left[\mathbf{y}_{1_{R}}^{\dagger}, \mathbf{y}_{2_{R}}^{\dagger}, \cdots, \mathbf{y}_{K_{R}}^{\dagger}\right]^{\dagger} \in \mathbb{C}^{N_{R}^{R x} \times 1}$, can be expressed as

$$
\mathbf{y}_{R}=\sum_{\ell_{U} \in \mathscr{U}} \mathbf{H}_{R, \ell_{U}} \mathbf{F}_{\ell_{U}} \mathbf{s}_{\ell_{U}}+\mathbf{G}_{R, R}\left(\tilde{\mathbf{x}}_{D}+\mathbf{q}_{D}\right)+\mathbf{q}_{U}+\mathbf{n}_{R} .
$$

Similar to $[24,52]$, the optimal minimum mean square error and successive interference cancellation (MMSE-SIC) technique is used at the CU to decode the UL messages. Based on the MMSE-SIC decoding strategy, the user's message with the strongest channel condition is retrieved first whereas that with the worst channel link is decoded last. After detecting any user data, the CU then removes it from the received signal for interference mitigation. As the result, the later decoding ULU suppresses from the multiple-access interferences of all earlier decoding ULUs. Therefore, the data rate of $\operatorname{ULU} \ell_{U}$ is given as $[14,24]$

$$
R_{\ell_{U}}=\log _{2}\left|\mathbf{I}_{N_{R}^{R x}}+\boldsymbol{\Omega}_{\ell_{U}}^{-1} \mathbf{H}_{R, \ell_{U}} \mathbf{F}_{\ell_{U}} \mathbf{F}_{\ell_{U}}^{\dagger} \mathbf{H}_{R, \ell_{U}}^{\dagger}\right|,
$$

where $\boldsymbol{\Omega}_{\ell_{U}}=\sum_{t_{U}>\ell_{U}}^{J_{U}} \mathbf{H}_{R, t_{U}} \mathbf{F}_{t_{U}} \mathbf{F}_{t_{U}}^{\dagger} \mathbf{H}_{R, t_{U}}^{\dagger}+\mathbf{G}_{R, R}\left(\sum_{k_{D} \in \mathscr{D}} \mathbf{F}_{k_{D}} \mathbf{F}_{k_{D}}^{\dagger}+\right.$ $\left.\mathbf{Q}_{D}\right) \mathbf{G}_{R, R}^{\dagger}+\mathbf{Q}_{U}+\sigma_{R}^{2} \mathbf{I}_{N_{R}^{R x}}$. Then, the total achievable rate of UL channels, i.e., $\sum_{\ell_{U} \in \mathcal{U}} R_{\ell_{U}}$, can be formulated as

$$
R_{R}=\log _{2}\left|\mathbf{I}_{N_{R}^{R x}}+\mathbf{\Omega}_{R}^{-1} \mathbf{C}_{R}\right|
$$

where $\mathbf{C}_{R}=\sum_{\ell_{U} \in \mathscr{U}} \mathbf{H}_{R, \ell_{U}} \mathbf{F}_{\ell_{U}} \mathbf{F}_{\ell_{U}}^{\dagger} \mathbf{H}_{R, \ell_{U}}^{\dagger}$ is the received covariance matrix of the desired UL signal at RUs. Meanwhile, the covariance matrix of SI and compression noise plus additive noise at RUs is

$$
\boldsymbol{\Omega}_{R}=\mathbf{G}_{R, R}\left(\sum_{k_{D} \in \mathscr{D}} \mathbf{F}_{k_{D}} \mathbf{F}_{k_{D}}^{\dagger}+\mathbf{Q}_{D}\right) \mathbf{G}_{R, R}^{\dagger}+\mathbf{Q}_{U}+\sigma_{R}^{2} \mathbf{I}_{N_{R}^{R x}},
$$

with $\mathbf{Q}_{U} \triangleq \operatorname{diag}\left\{\mathbf{Q}_{U, \lambda_{R}}, \lambda_{R} \in \mathscr{R}\right\}$ being the diagonally stacking matrix of covariance compression noise of UL FH links.

2.3. Power Consumption Model. The whole transmit power consumed by RUs and ULUs is given by

$$
\begin{aligned}
P_{\text {Tot }}^{T x}= & \sum_{\lambda_{R} \in \mathscr{R}} \xi_{\lambda_{R}}^{-1} P_{\lambda_{R}}^{T x}+\sum_{\ell_{U} \in \mathscr{U}} \xi_{\ell_{U}}^{-1} P_{\ell_{U}}^{T x}=\sum_{\lambda_{R} \in \mathscr{R}} \xi_{\lambda_{R}}^{-1}\left(\sum_{k_{D} \in \mathscr{D}}\left\|\mathbf{E}_{\lambda_{R}}^{\dagger} \mathbf{F}_{k_{D}}\right\|^{2}+\operatorname{Tr}\left(\mathbf{Q}_{D, \lambda_{R}}\right)\right) \\
& +\sum_{\ell_{U} \in \mathscr{U}} \xi_{\ell_{U}}^{-1}\left\|\mathbf{F}_{\ell_{U}}\right\|^{2},
\end{aligned}
$$

where $P_{\ell_{U}}^{T x}$ and $P_{\lambda_{R}}^{T x}$ are the transmitted power at ULU $\ell_{U}$ and RU $\lambda_{R}$ and given in (1) and (4), respectively. In addition, 0 $<\xi_{\lambda_{R}}, \xi_{\ell_{U}} \leq 1$ are the power amplifier efficiency factors of $\mathrm{RU} \lambda_{R}$ and ULU $\ell_{U}$, respectively. Otherwise, the total circuitry power is given as

$$
P_{\text {Tot }}^{C i r}=\sum_{\lambda_{R} \in \mathscr{R}} P_{\lambda_{R}}^{C i r}+\sum_{\ell_{U} \in \mathscr{U}} P_{\ell_{U}}^{C i r},
$$

where $P_{\lambda_{R}}^{C i r}$ and $P_{\ell_{U}}^{C i r}$ are the circuitry power consumed at RU $\lambda_{R}$ and ULU $\ell_{U}$, respectively. Additionally, the power consumption of the FH links is defined as the rate-dependent model; hence, the total power consumption over all DL and $\mathrm{UL}$ FH links is given as $[30,31]$

$$
P_{T o t}^{\mathrm{FH}}=\sum_{\lambda_{R} \in \mathscr{R}} \alpha_{U, \lambda_{R}}^{\mathrm{FH}} g_{U, \lambda_{R}}+\alpha_{D, \lambda_{R}}^{\mathrm{FH}} g_{D, \lambda_{R}}
$$

where $\alpha_{U, \lambda_{R}}^{\mathrm{FH}}=P_{U, \lambda_{R}, \max }^{\mathrm{FH}} C_{U, \lambda_{R}}^{-1}$ and $\alpha_{D, \lambda_{R}}^{\mathrm{FH}}=P_{D, \lambda_{R}, \max }^{\mathrm{FH}} C_{D, \lambda_{R}}^{-1}$ are constant scaling factors. $g_{D, \lambda_{R}}$ and $g_{U, \lambda_{R}}$ are FH rates given by (5) and (14), respectively. Meanwhile, $C_{D, \lambda_{R}}$ and $C_{U, \lambda_{R}}$ are the maximum rate to be able to convey through the DL and UL FH link $\lambda_{R}$, respectively. Nevertheless, $P_{U, \lambda_{R}, \max }^{\mathrm{FH}}$ and 
$P_{D, \lambda_{R}, \max }^{\mathrm{FH}}$ are the whole power consumption values when the FH link is active at the maximum rate [30, 31].

2.4. Formula of Considered Optimization Problems. Firstly, we introduce some system requirements including minimum harvested energy, minimum user's data rate, and limited $\mathrm{FH}$ capacity requirements. In order to ensure the operating necessity of the energy harvester, the total amount of harvested energy at DLUs must satisfy the following condition

$$
E_{k_{D}}^{\mathrm{NL}} \geq \bar{e}_{k_{D}}^{\mathrm{req}}, \mathbf{W}_{I, k_{D}} \geq \mathbf{0}_{N_{k_{D}}^{R x}}, \mathbf{I}_{N_{k_{D}}^{R x}} \geq \mathbf{W}_{I, k_{D}}, \quad \forall k_{D} \in \mathscr{D},
$$

where $\bar{e}_{k_{D}}^{\text {req }}$ is the required harvested energy at DLU $k_{D}$. The value of $\bar{e}_{k_{D}}^{\text {req }}$ is chosen properly providing that the harvested energy is useful and can activate the EH circuitry [50]. Hence, this value should be set up to be larger than or at least equal to the minimum sensitive level of its energy harvester $[26,39,48]$.

On the other hand, to meet the maximum limited $\mathrm{FH}$ capacity, the DL and UL FH rates are thus constrained as $[24,25]$

$$
\begin{gathered}
g_{D, \lambda_{R}} \leq C_{D, \lambda_{R}}, \quad \forall \lambda_{R} \in \mathscr{R}, \\
g_{U, \lambda_{R}} \leq C_{U, \lambda_{R}}, \quad \forall \lambda_{R} \in \mathscr{R} .
\end{gathered}
$$

Finally, the QoS management is predefined as the following achievable data rate constraints:

$$
\begin{gathered}
R_{k_{D}} \geq \bar{r}_{k_{D}}^{\mathrm{req}}, \quad \forall k_{D} \in \mathscr{D}, \\
R_{R} \geq \bar{r}_{R}^{\mathrm{req}},
\end{gathered}
$$

where $\bar{r}_{k_{D}}^{\text {req }}$ and $\bar{r}_{R}^{\text {req }}$ denote the minimum QoS requirement of DL and UL data rates, respectively.

For notation simplicity, we define the compact set $\delta$ as

$$
\mathcal{S} \triangleq\left\{\mathbf{Q}_{D, \lambda_{R}}, \mathbf{Q}_{U, \lambda_{R}}, \mathbf{F}_{k_{D}}, \mathbf{F}_{\ell_{U}}, \mathbf{W}_{k_{D}} \mid \quad k_{D} \in \mathscr{D}, \ell_{U} \in \mathscr{U}, \lambda_{R} \in \mathscr{R}\right\} .
$$

In this paper, we consider three SOOPs and an MOOP which are defined below.

2.4.1. Problem SOOP-1. The sum rate maximization problem is written as a minimization one

$$
\begin{gathered}
\min _{\mathcal{S}} F_{1}(\mathcal{S}) \triangleq-\mathscr{R}_{\mathrm{Tot}}(\mathcal{S}) \\
\text { s.t.(1), (4), (23), (24), (25), (26), (27), }
\end{gathered}
$$

where $\mathscr{R}_{T o t}(\mathcal{S}) \triangleq \sum_{k_{D} \in \mathscr{D}} R_{k_{D}}+R_{R}$ is the overall UL and DL rate.

2.4.2. Problem SOOP-2. The power consumption minimization problem can be expressed as

$$
\min _{\mathcal{S}} F_{2}(\mathcal{S}) \triangleq \mathscr{P}_{T o t}(\mathcal{S})
$$

$$
\text { s.t.(1), (4), (23), (24), (25), (26), (27), }
$$

where $\mathscr{P}_{\text {Tot }}(\mathcal{S})=P_{\text {Tot }}^{T x}+P_{\text {Tot }}^{F H}+P_{\text {Tot }}^{C i r}$ is the whole power consumed for data transmission, FH links, and circuitry.

2.4.3. Problem SOOP-3. The conventional EE maximization problem is

$$
\begin{gathered}
\max _{\mathcal{S}} \mathscr{E}_{\text {Tot }}(\mathcal{S}) \\
\text { s.t.(1), (4), (23), (24), (25), (26), (27), }
\end{gathered}
$$

where $\mathscr{E}_{\text {Tot }}(\mathcal{S}) \triangleq \mathscr{R}_{\text {Tot }}(\mathcal{S}) / \mathscr{P}_{\text {Tot }}(\mathcal{S})$ is the whole system EE.

Note that (1) and (4) present the transmit power constraints at ULUs and RUs, respectively. The nonconvex constraints in (23) guarantee the minimum harvested energy at each EH-DLU while the nonconvex constraints of DL and UL FH rate are given in (24) and (25), respectively. As a result, the three above problems are highly nonconvex due not only to nonconvexities in objective functions but also to nonconvex constraints. Thus, these problems are all extremely intractable to directly find their own optimum points. In the following sections, we develop the iterative algorithms using sequential convex approximation (SCA) methods to solve these nonconvex problems.

2.4.4. Problem MOOP. The energy-spectral efficiency optimization using the MOO framework is given as $[37,38]$

$$
\min _{\mathcal{S}}\left[F_{1}(\mathcal{S}), F_{2}(\mathcal{S})\right]^{T}
$$

$$
\text { s.t.(1), (4), (23), (24), (25), (26), (27), }
$$

in which the objective function is a two-row vector whose elements are the SOOP-1 and SOOP-2 objective functions. Once again, it is very challenging to jointly find the optimal solution to these nonconvex multicriteria problems (32a) and (32b). Therefore, in the next section, we introduce an approach based on the modified weighted Tchebycheff and SCA methods to find the optimal Pareto set.

\section{Proposed Iterative Algorithm}

This section represents an approach to develop algorithms for considered nonconvex problems using SCA methods. Firstly, we provide three inner convex approximations of the data rate, $\mathrm{FH}$ rate, and harvested energy.

3.1. Convex Inner Approximation Analysis. In this subsection, we provide inner approximations in order to convexify the nonconvex problems.

3.1.1. Concave Quadratic Lower Approximation for the $D L$ and UL Rate. Following Theorem 1 of [52], [53], and Appen$\operatorname{dix}$ A that the DLU $k_{D}$ rate in (9) is lower bounded by the following concave quadratic expression, 


$$
\begin{aligned}
\tilde{R}_{k_{D}}= & a_{k_{D}}^{(\kappa)}+\frac{2}{\ln (2)} \mathfrak{R}\left\{\operatorname{Tr}\left(\mathscr{A}_{k_{D}}^{(\kappa)} \mathbf{F}_{k_{D}}\right)\right\}-\frac{1}{\ln (2)} \\
& \times \operatorname{Tr}\left(\mathscr{B}_{k_{D}}^{(\kappa)}\left(\boldsymbol{\Omega}_{k_{D}}+\mathbf{C}_{k_{D}}+\sigma_{k_{D}, c}^{2} \mathbf{W}_{k_{D}}^{-1}\right)\right),
\end{aligned}
$$

where $\boldsymbol{\Phi}_{k_{D}}^{(\kappa)}=\boldsymbol{\Omega}_{k_{D}}^{(\kappa)}+\sigma_{k_{D}, c}^{2} \mathbf{W}_{k_{D}}^{(\kappa),-1}$ and

$$
\begin{gathered}
a_{k_{D}}^{(\kappa)}=\log _{2}\left|\mathbf{I}_{N_{k_{D}}^{k x}}+\boldsymbol{\Phi}_{k_{D}}^{(\kappa),-1} \mathbf{C}_{k_{D}}^{(\kappa)}\right|-\frac{1}{\ln (2)} \operatorname{Tr}\left(\boldsymbol{\Phi}_{k_{D}}^{(\kappa),-1} \mathbf{C}_{k_{D}}^{(\kappa)}\right) \leq 0, \\
\mathscr{A}_{k_{D}}^{(\kappa)}=\mathbf{F}_{k_{D}}^{(\kappa), \dagger} \mathbf{H}_{k_{D}}^{\dagger} \boldsymbol{\Phi}_{k_{D}}^{(\kappa),-1} \mathbf{H}_{k_{D}}, \\
\mathscr{B}_{k_{D}}^{(\kappa)}=\boldsymbol{\Phi}_{k_{D}}^{(\kappa),-1}-\left(\boldsymbol{\Phi}_{k_{D}}^{(\kappa)}+\mathbf{C}_{k_{D}}^{(\kappa)}\right)^{-1} \geq 0 .
\end{gathered}
$$

Moreover, the concave quadratic lower bound of UL rate (18) is given as

$$
\begin{aligned}
\tilde{R}_{R}= & a_{R}^{(\kappa)}+\frac{2}{\ln (2))} \mathfrak{R}\left\{\operatorname{Tr}\left(\mathscr{A}_{R}^{(\kappa)} \mathbf{F}_{U}\right)\right\}-\frac{1}{\ln (2)} \\
& \times \operatorname{Tr}\left(\mathscr{B}_{R}^{(\kappa)} \mathbf{\Omega}_{R}+\mathscr{B}_{R}^{(\kappa)} \mathbf{C}_{R}\right),
\end{aligned}
$$

where $\mathbf{H}_{R, U} \triangleq\left[\mathbf{H}_{R, 1_{U}}, \mathbf{H}_{R, 2_{U}}, \cdots \mathbf{H}_{R, K_{U}}\right], \mathbf{F}_{U} \triangleq \operatorname{diag}\left\{\mathbf{F}_{\ell_{U}}, \ell_{U} \in \mathscr{U}\right.$ \},$d_{U}=\sum_{\ell_{U} \in \mathcal{U}} d_{\ell_{U}}$, and

$$
\begin{gathered}
a_{R}^{(\kappa)}=\log _{2}\left|\mathbf{I}_{N_{R}^{R x}}+\boldsymbol{\Omega}_{R}^{(\kappa),-1} \mathbf{C}_{R}^{(\kappa)}\right|-\frac{1}{\ln (2)} \operatorname{Tr}\left(\boldsymbol{\Omega}_{R}^{(\kappa),-1} \mathbf{C}_{R}^{(\kappa)}\right) \leq 0, \\
\mathscr{A}_{R}^{(\kappa)}=\mathbf{F}_{U}^{(\kappa), \dagger} \mathbf{H}_{R, U}^{\dagger} \mathbf{\Omega}_{R}^{(\kappa),-1} \mathbf{H}_{R, U}, \\
\mathscr{B}_{R}^{(\kappa)}=\boldsymbol{\Omega}_{R}^{(\kappa),-1}-\left(\boldsymbol{\Omega}_{R}^{(\kappa)}+\mathbf{C}_{R}^{(\kappa)}\right)^{-1} \geq 0 .
\end{gathered}
$$

It is shown in $[52$

that-

$\tilde{R}_{k_{D}} \leq R_{k_{D}}, \tilde{R}_{R} \leq R_{R}, \forall \mathcal{S}$, and $\tilde{R}_{k_{D}}=R_{k_{D}}, \tilde{R}_{R}=R_{R}$, at $\mathcal{S} \equiv \mathcal{S}^{(\kappa)}$

Accordingly, the nonconvex QoS rate constraints (26) and (27) are innerly approximated, respectively, as the following convex constraints

$$
\begin{gathered}
\tilde{R}_{k_{D}} \geq \bar{r}_{k_{D}}^{\text {req }}, \quad \forall k_{D} \in \mathscr{D}, \\
\tilde{R}_{R} \geq \bar{r}_{R}^{\text {req }} .
\end{gathered}
$$

3.1.2. Convex Inner Approximation for DL and UL FH Rate Constraint. The convex upper bound of the DL FH rate is given by

$$
\widehat{\mathfrak{g}}_{D, \lambda_{R}}=g_{D, \lambda_{R}}^{(\kappa)}+\frac{1}{\ln (2)} \operatorname{Tr}\left(\mathbf{Q}_{D, \lambda_{R}}^{(\kappa)} \mathbf{Q}_{D, \lambda_{R}}^{-1}+\boldsymbol{\Theta}_{\lambda_{R}}^{(\kappa),-1} \boldsymbol{\Theta}_{\lambda_{R}}\right) \geq g_{D, \lambda_{R}},
$$

where $g_{D, \lambda_{R}}^{(\kappa)}=\log _{2}\left|\mathbf{Q}_{D, \lambda_{R}}^{(\kappa),-1} \boldsymbol{\Theta}_{\lambda_{R}}^{(\kappa)}\right|-2 N_{\lambda_{R}}^{T x} / \ln (2)$ with

$$
\begin{gathered}
\boldsymbol{\Theta}_{\lambda_{R}}=\sum_{k_{D} \in \mathscr{D}} \mathbf{E}_{\lambda_{R}}^{\dagger} \mathbf{F}_{k_{D}} \mathbf{F}_{k_{D}}^{\dagger} \mathbf{E}_{\lambda_{R}}+\mathbf{Q}_{D, \lambda_{R}}, \\
\boldsymbol{\Theta}_{\lambda_{R}}^{(\kappa)}=\sum_{k_{D} \in \mathscr{D}} \mathbf{E}_{\lambda_{R}}^{\dagger} \mathbf{F}_{k_{D}}^{(\kappa)} \mathbf{F}_{k_{D}}^{(\kappa), \dagger} \mathbf{E}_{\lambda_{R}}+\mathbf{Q}_{D, \lambda_{R}}^{(\kappa)} .
\end{gathered}
$$

In addition, the UL FH rate is convex upper bounded by

$$
\begin{aligned}
\widehat{\mathcal{g}}_{U, \lambda_{R}}= & g_{U, \lambda_{R}}^{(\kappa)}+\frac{1}{\ln (2)} \operatorname{Tr}\left(\mathbf{Q}_{U, \lambda_{R}}^{(\kappa)} \mathbf{Q}_{U, \lambda_{R}}^{-1}\right)+\frac{1}{\ln (2)} \\
& \times \operatorname{Tr}\left(\left(\mathbf{Q}_{U, \lambda_{R}}^{(\kappa)}+\mathbf{\Xi}_{U, \lambda_{R}}^{(\kappa)}\right)^{-1}\left(\mathbf{Q}_{U, \lambda_{R}}+\boldsymbol{\Xi}_{U, \lambda_{R}}\right)\right) \geq \mathfrak{g}_{U, \lambda_{R}},
\end{aligned}
$$

where $g_{U, \lambda_{R}}^{(\kappa)}=\log _{2}\left|\mathbf{I}_{N_{\lambda_{R}}^{R x}}+\mathbf{Q}_{U, \lambda_{R}}^{(\kappa),-1} \boldsymbol{\Xi}_{U, \lambda_{R}}^{(\kappa)}\right|-2 N_{\lambda_{R}}^{R x} / \ln (2)$, with $\mathbf{\Xi}_{U, \lambda_{R}}^{(\kappa)}$ being found by substituting $\mathbf{F}_{\ell_{U}}^{(\kappa)}, \mathbf{F}_{k_{D}}^{(\kappa)}$, and $\mathbf{Q}_{D, \theta_{R}}^{(\kappa)}$ into (15) instead. The complete proof is given in Appendix B.

Finally, the inner convex approximation of nonconvex $\mathrm{FH}$ rate constraints according to the DL and UL in (24) and (25), respectively, is given as

$$
\begin{gathered}
\widehat{g}_{D, \lambda_{R}} \leq C_{D, \lambda_{R}}, \\
\widehat{\mathcal{g}}_{U, \lambda_{R}} \leq C_{U, \lambda_{R}}, \\
\forall \lambda_{R} \in \mathscr{R} .
\end{gathered}
$$

3.1.3. Convex Inner Approximation for DLU EH Constraints. Firstly, the compact constraint in (23) is equivalently rewritten after some simple mathematical manipulations as

$$
E_{k_{D}} \geq e_{k_{D}}^{\text {req }}, \mathbf{W}_{I, k_{D}} \geq \mathbf{0}_{N_{k_{D}}^{R x}}, \mathbf{I}_{N_{k_{D}}^{R x}} \geq \mathbf{W}_{I, k_{D}}, \quad \forall k_{D} \in \mathscr{D},
$$

where $e_{k_{D}}^{\text {req }} \triangleq b_{k_{D}}-a_{k_{D}}^{-1} \ln \left(\left(e_{k_{D}}^{\text {req }} \Delta_{k_{D}}^{-1}+\Omega_{k_{D}}\right)^{-1}-1\right)$. Next, by introducing additional diagonal matrix variables $\mathbf{X}_{k_{D}}$ with $k_{D} \in \mathscr{D}$, which satisfies the constraints

$$
\mathbf{X}_{k_{D}} \succeq \mathbf{I}_{N_{k_{D}}^{R x}}, \mathbf{I}_{N_{k_{D}}^{R x}}-\mathbf{W}_{k_{D}} \succeq \mathbf{X}_{k_{D}}^{-1}, \quad \forall k_{D} \in \mathscr{D},
$$

the harvested energy at DLU $k_{D}$ is lower, approximated as (see Appendix C for detail)

$$
\begin{aligned}
E_{k_{D}} \geq & \widehat{E}_{k_{D}}=\sum_{i_{D} \in \mathscr{D}} \operatorname{Tr}\left(\varphi\left(\mathbf{H}_{k_{D}} \mathbf{F}_{i_{D}}, \mathbf{X}_{k_{D}}\right)\right)+\sum_{\ell_{U} \in \mathcal{U}} \operatorname{Tr}\left(\varphi\left(\mathbf{G}_{k_{D}, \ell_{U}} \mathbf{F}_{\ell_{U}}, \mathbf{X}_{k_{D}}\right)\right) \\
& -\vartheta\left(\mathbf{W}_{k_{D}}, \mathbf{H}_{k_{D}} \mathbf{Q}_{D} \mathbf{H}_{k_{D}}^{\dagger}\right)+\operatorname{Tr}\left(\mathbf{H}_{k_{D}} \mathbf{Q}_{D} \mathbf{H}_{k_{D}}^{\dagger}\right)+\sigma_{k_{D}, a}^{2} \operatorname{Tr}\left(\mathbf{I}_{N_{k_{D}}^{R x}}-\mathbf{W}_{k_{D}}\right),
\end{aligned}
$$

with

$$
\begin{aligned}
\varphi\left(\mathbf{H}_{k_{D}} \mathbf{F}_{i_{D}}, \mathbf{X}_{k_{D}}\right)= & \left(\mathbf{H}_{k_{D}} \mathbf{F}_{i_{D}}^{(\kappa)}\right)^{\dagger} \mathbf{X}_{k_{D}}^{(\kappa),-1} \mathbf{H}_{k_{D}} \mathbf{F}_{i_{D}}+\left(\mathbf{H}_{k_{D}} \mathbf{F}_{i_{D}}\right)^{\dagger} \mathbf{X}_{k_{D}}^{(\kappa),-1} \mathbf{H}_{k_{D}} \mathbf{F}_{i_{D}}^{(\kappa)} \\
& +\left(\mathbf{H}_{k_{D}} \mathbf{F}_{i_{D}}^{(\kappa)}\right)^{\dagger} \mathbf{X}_{k_{D}}^{(\kappa),-1} \mathbf{X}_{k_{D}} \mathbf{X}_{k_{D}}^{(\kappa),-1} \mathbf{H}_{k_{D}} \mathbf{F}_{i_{D}}^{(\kappa)},
\end{aligned}
$$


Initialization: Setting the small error tolerance $\varepsilon>0$, the maximum number of iteration $\kappa_{\max }$. Generating an initial set $\tilde{\mathcal{S}}^{(0)}$ by solving max-min problem (58a) and (58b);

Set $\kappa=0$;

repeat

Find optimal set $\tilde{\mathcal{S}}^{\star}$ by solving convex problem (50a) and (50b) [or (51a) and (51b)],

Update $\kappa \leftarrow \kappa+1$ and $\tilde{\mathcal{S}}^{(\kappa)} \leftarrow \tilde{\mathcal{S}}^{\star}$;

until reaches $1-\tilde{F}_{k}^{(\kappa)} / \tilde{F}_{k}^{(\kappa-1)} \leq \varepsilon$ with $k=1$ [or $k=2$ ];

Output: The optimum point $\delta^{\text {opt }} \leftarrow \delta^{\star}$.

Algorithm 1: The iterative algorithm for the sum-SE maximization (or sum-power consumption minimization) problem.

$$
\begin{aligned}
\varphi\left(\mathbf{G}_{k_{D}, \ell_{U}} \mathbf{F}_{\ell_{U}}, \mathbf{X}_{k_{D}}\right)= & \left(\mathbf{G}_{k_{D}, \ell_{U}} \mathbf{F}_{\ell_{U}}^{(\kappa)}\right)^{\dagger} \mathbf{X}_{k_{D}}^{(\kappa),-1} \mathbf{G}_{k_{D}, \ell_{U}} \mathbf{F}_{\ell_{U}} \\
& +\left(\mathbf{G}_{k_{D}, \ell_{U}} \mathbf{F}_{\ell_{U}}\right)^{\dagger} \mathbf{X}_{k_{D},{ }^{(\kappa),-1}} \mathbf{G}_{k_{D}, \ell_{U}} \mathbf{F}_{\ell_{U}}^{(\kappa)} \\
& +\left(\mathbf{G}_{k_{D}, \ell_{U}} \mathbf{F}_{\ell_{U}}^{(\kappa)}\right)^{\dagger} \mathbf{X}_{k_{D}}^{(\kappa),-1} \mathbf{X}_{k_{D}} \mathbf{X}_{k_{D}}^{(\kappa),-1} \mathbf{G}_{k_{D}, \ell_{U}} \mathbf{F}_{\ell_{U}}^{(\kappa)}
\end{aligned}
$$

$$
\begin{aligned}
\vartheta\left(\mathbf{W}_{k_{D}}, \mathbf{H}_{k_{D}} \mathbf{Q}_{D} \mathbf{H}_{k_{D}}^{\dagger}\right)= & \frac{1}{2}\left\|\mathbf{W}_{k_{D}}^{(\kappa),-0.5} \mathbf{W}_{k_{D}}\left(\mathbf{H}_{k_{D}} \mathbf{Q}_{D}^{(\kappa)} \mathbf{H}_{k_{D}}^{\dagger}\right)^{0.5}\right\|^{2} \\
& +\frac{1}{2}\left\|\mathbf{W}_{k_{D}}^{(\kappa), 0.5} \mathbf{H}_{k_{D}} \mathbf{Q}_{D} \mathbf{H}_{k_{D}}^{\dagger}\left(\mathbf{H}_{k_{D}} \mathbf{Q}_{D}^{(\kappa)} \mathbf{H}_{k_{D}}^{\dagger}\right)^{-0.5}\right\|^{2} .
\end{aligned}
$$

Consequently, the nonconvex constraint (43) is recast as the following convex inner approximation below

$$
\begin{aligned}
& \widehat{E}_{k_{D}} \geq e_{k_{D}}^{\mathrm{req}}, \mathbf{W}_{I, k_{D}} \geq \mathbf{0}_{N_{k_{D}}^{R x}}, \mathbf{I}_{N_{k_{D}}^{R x}} \geq \mathbf{W}_{I, k_{D}}, \quad \forall k_{D} \in \mathscr{D} \\
& \quad: \text { (44). }
\end{aligned}
$$

3.2. Iterative Algorithm for Considered Optimization Problems. Upon the above analyses, the nonconvex SOOP-1 in (29a) is innerly approximated at iteration $\kappa$ as the following convex one

$$
\begin{gathered}
\min _{\tilde{\mathcal{S}}} \tilde{F}_{1}(\mathcal{S}) \triangleq-\tilde{\mathscr{R}}_{\text {Tot }}(\mathcal{S}) \\
\text { s.t.(1), (4), (37), (38), (42), (49), }
\end{gathered}
$$

where $\mathscr{R}_{\text {Tot }}(\mathcal{S}) \geq \tilde{\mathscr{R}}_{\text {Tot }}(\mathcal{S}) \triangleq \sum_{k_{D} \in \mathscr{D}} \tilde{R}_{k_{D}}+\tilde{R}_{R}$ and $\tilde{\mathcal{S}}=\{\mathcal{S}, \mathbf{X}\}$ with $\mathbf{X}=\left\{\mathbf{X}_{k_{D}} \mid k_{D} \in \mathscr{D}\right\}$. Additionally, the power consumption minimization problems (30a) and (30b) can be expressed as convex one at iteration $\kappa$

$$
\begin{gathered}
\min _{\tilde{\delta}} \tilde{F}_{2}(\mathcal{S}) \triangleq \tilde{\mathscr{P}}_{\text {Tot }}(\mathcal{S}) \\
\text { s.t.(1), (4), (37), (38), (42), (49), }
\end{gathered}
$$

where $\tilde{\mathscr{P}}_{\text {Tot }}(\mathcal{S}) \geq \mathscr{P}_{\text {Tot }}(\mathcal{S})$ and given by

$$
\tilde{\mathscr{P}}_{\text {Tot }}(\mathcal{S})=P_{\text {Tot }}^{T x}+\sum_{\lambda_{R} \in \mathscr{R}}\left(\alpha_{U, \lambda_{R}}^{\mathrm{FH}} \widehat{g}_{U, \lambda_{R}}+\alpha_{D, \lambda_{R}}^{\mathrm{FH}} \widehat{g}_{D, \lambda_{R}}\right)+P_{\text {Tot }}^{C i r} \text {, }
$$

where $\widehat{g}_{U, \lambda_{R}}$ and $\widehat{g}_{D, \lambda_{R}}$ are given in (39) and (41), respectively.

These two recast problems are now convex ones and can be efficiently tackled with existing convex solvers, such as CVX packages [54]. The whole procedure is summarized in Algorithm 1.

On the other hand, in order to find the Pareto set, the MOO problems (32a) and (32b) are first transformed to the SOO one by utilizing the modified weighted Tchebycheff method as [37]

$$
\begin{aligned}
& \min _{\mathcal{S}} \max _{p=1,2} \omega_{p}\left[\frac{F_{p}(\mathcal{S})-F_{p}^{\star}}{\left|F_{p}^{\star}\right|}+\delta \sum_{q=1}^{2} \frac{F_{q}(\mathcal{S})-F_{q}^{\star}}{\left|F_{q}^{\star}\right|}\right] \\
& \text { s.t.(1), (4), (23), (24), (25), (26), (27), }
\end{aligned}
$$

where $F_{1}^{\star}$ and $F_{2}^{\star}$ are the optimums of SOOP-1 and SOOP-2, respectively. The weighted factor $\omega_{p} \in[0,1]$ with $\omega_{1}+\omega_{2}=1$ represents the trade-off between two considered metrics. In addition, the small constant $\delta=[0.001,0.01]$ should be chosen to obtain the Pareto optimality $[19,37]$.

It is important to notice that problems (53a) and (53b) are still challenging to be directly solved due to the nonsmooth nonconvex property of the objective function and nonconvex constraints. Therefore, we exploit the analyses in the previous subsections and SCA method to develop an algorithm to iteratively handle it. Following inner convex approximations in the previous subsection, problems (53a) and (53b) are approximated at iteration $\kappa$ as

$$
\begin{gathered}
\min _{\tilde{\delta}} \max _{p=1,2} \omega_{p}\left[\frac{\tilde{F}_{p}(\mathcal{S})-F_{p}^{\star}}{\left|F_{p}^{\star}\right|}+\delta \sum_{q=1}^{2} \frac{\tilde{F}_{q}(\mathcal{S})-F_{q}^{\star}}{\left|F_{q}^{\star}\right|}\right] \\
\text { s.t. (1), (4), (37), (38), (42), (49), }
\end{gathered}
$$

For given $\omega_{1}$ and $\omega_{2}$, problems (54a) and (54b) are a convex one and can be handled with existing convex solvers, e.g., 
Initialization: Set the error tolerance $\varepsilon>0$, the maximum number of iteration $\kappa_{\max }$, maximum $\rho_{\max }$ and $\rho=0$; Set weighted parameter sets $\omega_{1}=\left[\omega_{1}^{(1)}, \omega_{1}^{(2)}, \cdots, \omega_{1}^{\left(\rho_{\max }\right)}\right]$ and $\omega_{2}=1-\omega_{1}$;

Generating initial set of $\tilde{\mathcal{S}}^{(0)}$ by solving max-min problem (58a) and (58b);

Find the optimum $F_{1}^{\star}$ and $F_{2}^{\star}$ by solving (29a), (29b), (30a), and (30b), respectively, with Algorithm 1;

repeat

Update $\rho \leftarrow \rho+1$; Set $\kappa=0$;

repeat

Given $\omega_{1}^{(\rho)}, \omega_{2}^{(\rho)}$, find the optimal set $\tilde{\mathcal{S}}^{\star}$ by solving convex problem (54a) and (54b);

Update $\kappa \leftarrow \kappa+1$ and $\tilde{\mathcal{S}}^{(\kappa)} \leftarrow \tilde{\mathcal{S}}^{\star}$;

Compute $\Delta \eta=1-\eta^{(\kappa)} / \eta^{(\kappa-1)}$ with $\eta$ is objective value in (54a);

until reaches $|\Delta \eta| \leq \varepsilon$ or $\kappa=\kappa_{\max }$;

Update the $\rho$-th component of the Pareto set: $\mathcal{S}_{(\rho)}^{\mathrm{opt}} \leftarrow \mathcal{S}^{*}$;

until reaches $\rho=\rho_{\text {max }}$;

Output: The optimal Pareto set $\left\{\mathcal{S}_{(\rho)}^{\text {opt }}: \rho=1,2, \cdots, \rho_{\max }\right\}$.

Algorithm 2: Iterative algorithm for finding the optimal Pareto set.

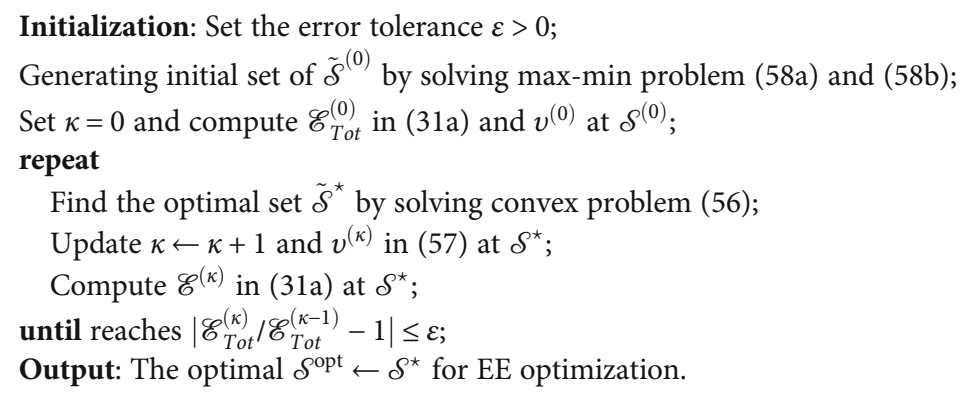

Algorithm 3: The Dinkelbach approach-based iterative algorithm for EE optimization.

CVX packages [54]. The entire set of optimal Pareto points is achieved by varying value $0 \leq \omega_{1}, \omega_{2} \leq 1$ under the condition of $\omega_{1}+\omega_{2}=1$. The total procedure to find Pareto points is summarized in Algorithm 2.

\subsection{Dinkelbach-Based Iterative Algorithm for the Conventional} Fractional EE Maximization Problem. Next, we turn our attention to the traditional fractional EE optimization problem. With the bound expressions derived in the previous subsection, the EE maximization problem can be recast at iteration $\kappa$ as

$$
\begin{gathered}
\max _{\tilde{\mathcal{S}}} \tilde{\mathscr{E}}_{T o t}(\mathcal{S}) \triangleq \frac{\tilde{\mathscr{R}}_{T o t}(\mathcal{S})}{\tilde{\mathscr{P}}_{T o t}(\mathcal{S})} \\
\text { s.t.(1), (4), (37), (38), (42), (49), }
\end{gathered}
$$

$(\mathcal{S})$ being already defined below as (50a), (50b), (51a), and (51b), respectively. Problems (55a) and (55b) are now a concave-convex fractional program with convex constraints. In specific, the objective is a pseudoconcave function that inspires us to exploit the nonlinear fractional programming approach [55]. Based on the Dinkelbach algorithm, we first reformulate (55a) and (55b) into a parameterized polynomial subtractive form with respect to $v$ as follows

$$
G(v)= \begin{cases}\max _{\{\tilde{\mathcal{S}}, \mathbf{X}\}} & \tilde{\mathscr{R}}_{\text {Tot }}(\mathcal{S})-v \tilde{\mathscr{P}}_{\text {Tot }}(\mathcal{S}), \\ \text { s.t. } & (1),(4),(37),(38),(42),(49),\end{cases}
$$

Following [56], it is emphasized on parametrized problem (56) that $G(v)$ is a monotonically decreasing and continuous function with respect to $v$. This implies that $G(v)=0$ is fulfilled with a unique solution which is given as

$$
v^{\star}=\tilde{\mathscr{E}}^{\star}
$$

On the other hand, the optimal solution of (56) at $v^{\star}$ is also an optimal solution of (55a) and (55b) and $v^{\star}$ is the optimal objective value. The detailed explanation can be refereed in [56]. It implies that one can first fix $v$ to find the optimal solution of convex problems (55a) and (55b). Afterward, we tackle identity $G(v)=0$ to get the unique $v^{\star}$. Finally, the whole procedure to achieve the optimal solution is summarized in Algorithm 3. 


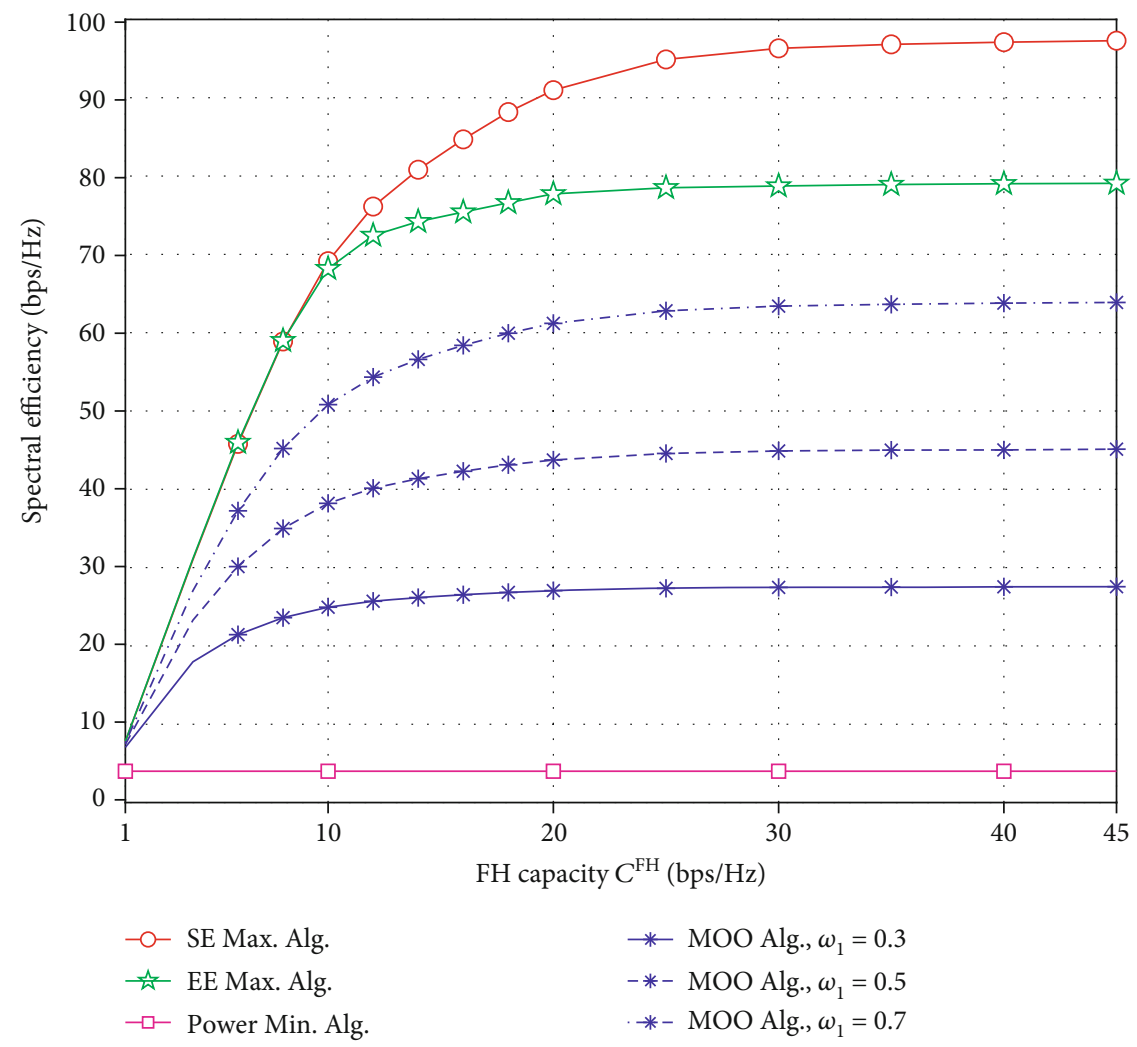

FIgURE 2: The average system SE versus the capable FH capacity with various design methods.

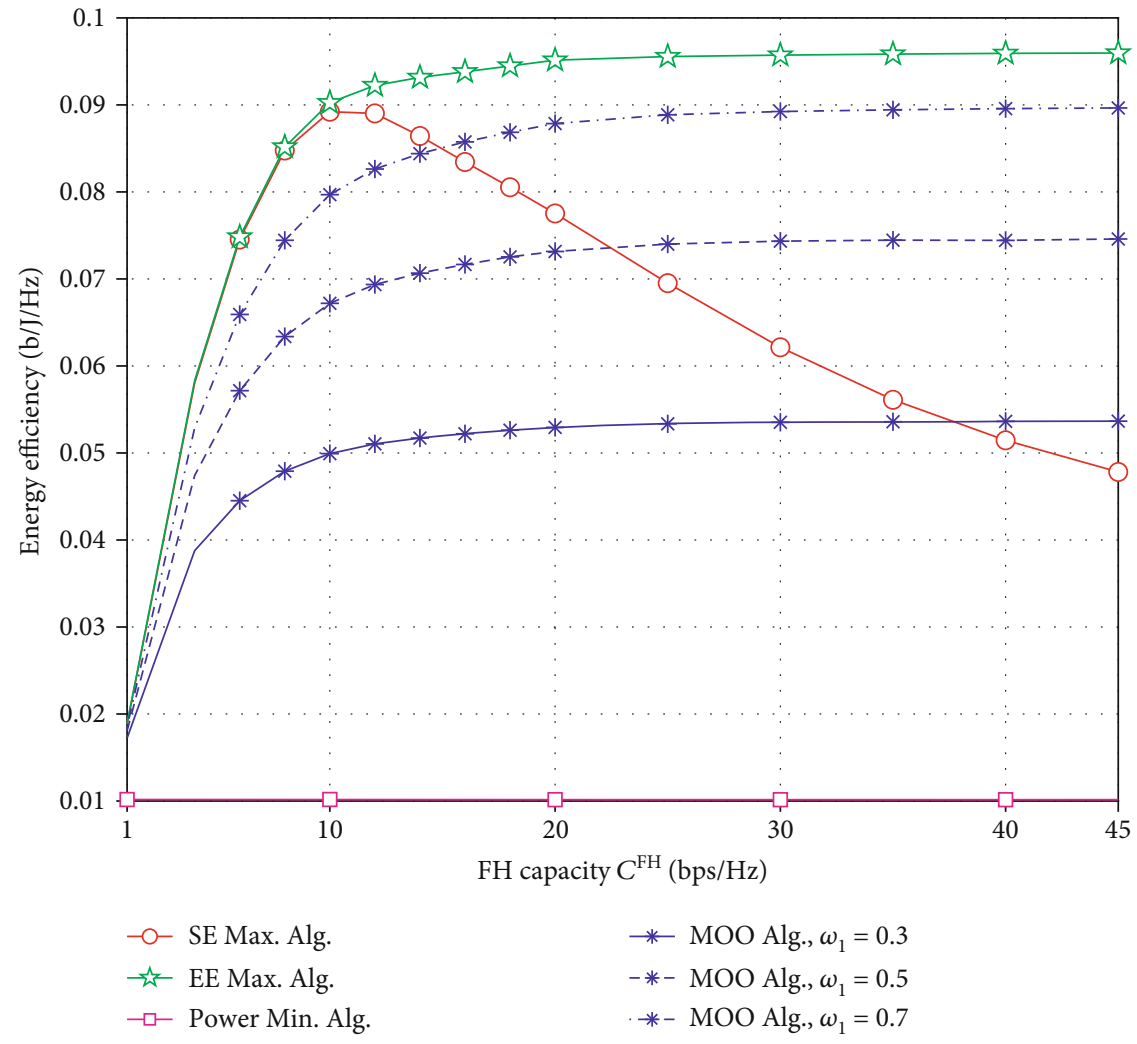

FIgURE 3: The average system EE versus the capable FH capacity with various design methods. 


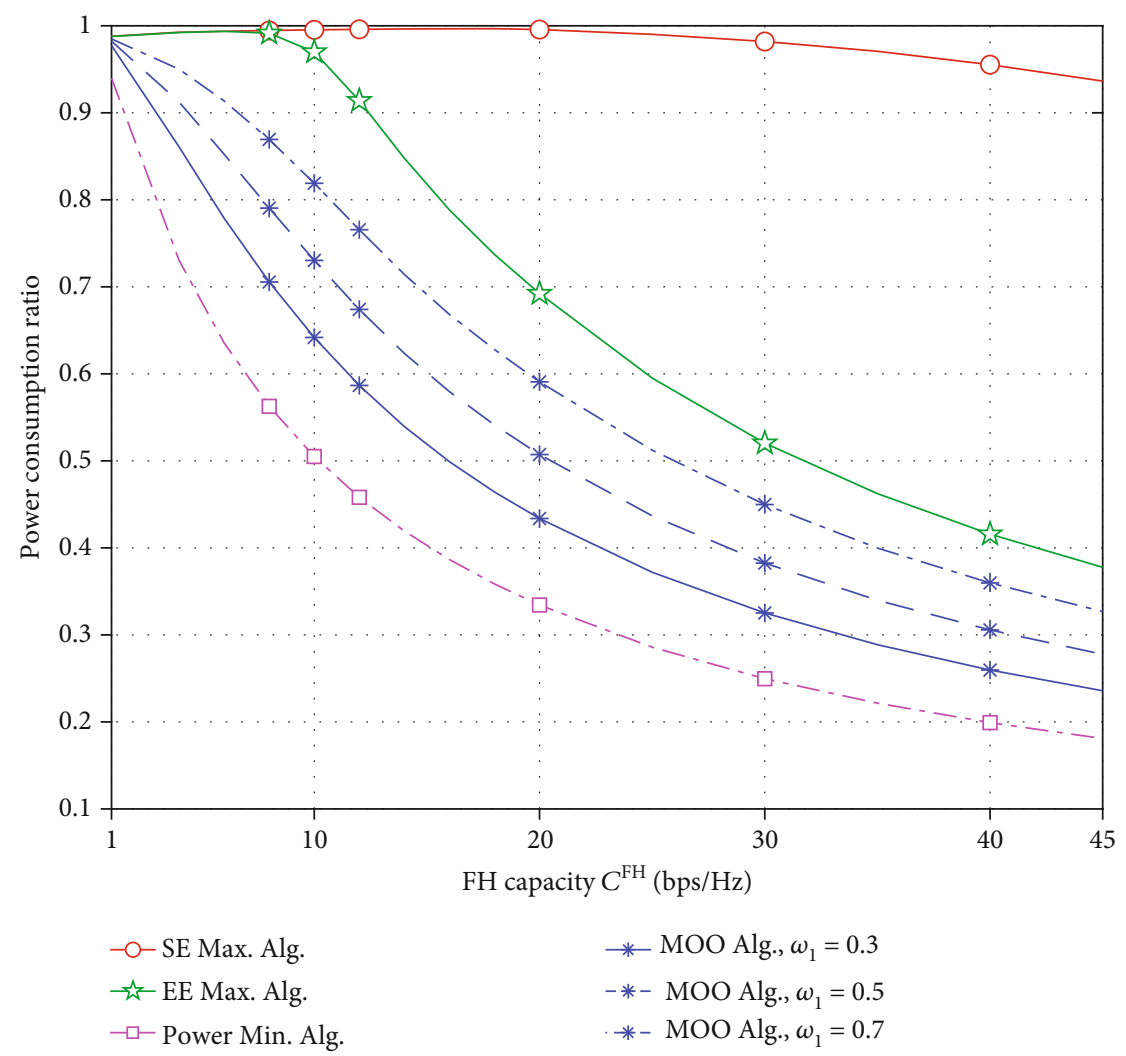

FIGURE 4: The ratio between the total consumed power to the system power budget versus the capable FH capacity with various design methods.

3.4. Initiating a Feasible Point. At the beginning of the proposed algorithms, it is hard to find one feasible set. Thus, we consider the following max-min problem which helps to find an initial feasible set. The initial feasible point is generated by solving the following max-min problem

$$
\begin{aligned}
& \max _{\mathcal{S}} \min _{k_{D} \in \mathscr{D}}\left(\frac{E_{k_{D}}}{e_{k_{D}}^{\min }}, \frac{R_{k_{D}}}{\bar{r}_{k_{D}}^{\mathrm{req}}}, \frac{R_{R}}{\bar{r}_{R}^{\mathrm{req}}}\right) \\
& \text { s.t. }(1),(4),(24),(25) .
\end{aligned}
$$

The solution can be iteratively found by solving a inner convex approximation problem at iteration $\kappa$ as

$$
\begin{gathered}
\max _{\mathcal{S}} \min _{k_{D} \in \mathscr{D}}\left(\frac{\widehat{E}_{k_{D}}}{e_{k_{D}}^{\min }}, \frac{\tilde{R}_{k_{D}}}{\bar{r}_{k_{D}}^{\mathrm{req}}}, \frac{\tilde{R}_{R}}{\bar{r}_{R}^{\mathrm{req}}}\right) \\
\text { s.t. }(1),(4),(42),(44)
\end{gathered}
$$

where $\tilde{R}_{k_{D}}, \tilde{R}_{R}$, and $\widehat{E}_{k_{D}}$ are given in (33), (35), and (45), respectively. The algorithm terminates as the objective value is larger than or equal to 1 .

\section{Numerical Results}

In this section, various numerical simulation results are conducted to illustrate EE-SE trade-offs for the interested FD MU-MIMO CRANs with SWIPT.

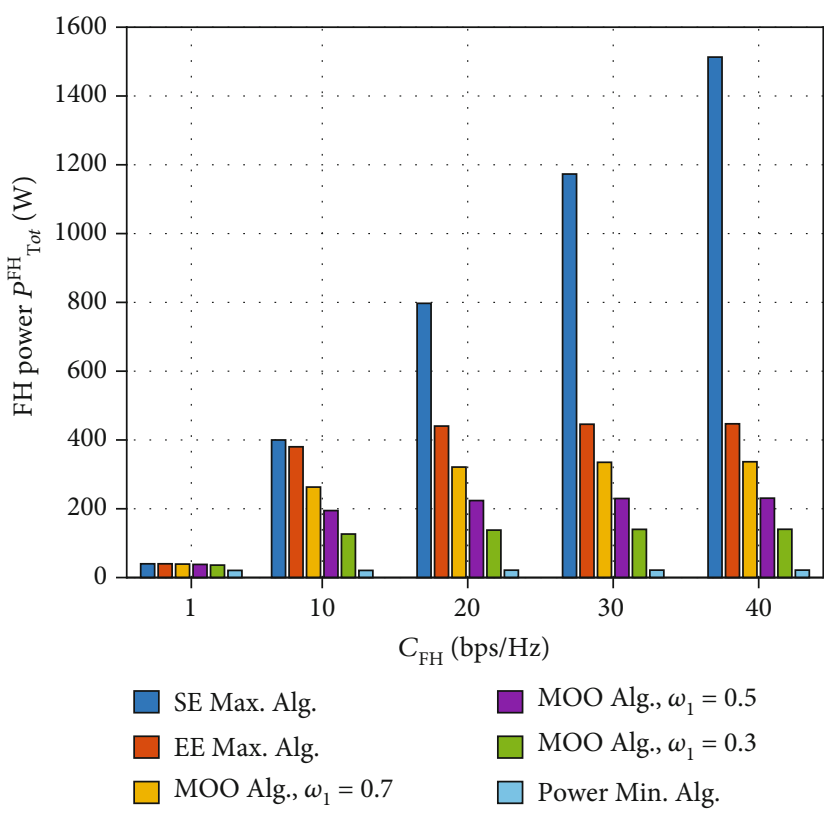

FIgURE 5: The average FH power with various FH capacity levels and design methods.

4.1. Simulation Parameters. We adopt the CRAN model in $[24,25]$, in which a square cell with a length of 100 meters is divided into four equally small square cells with a length of 50 meters. One RU is located at the center of each small 


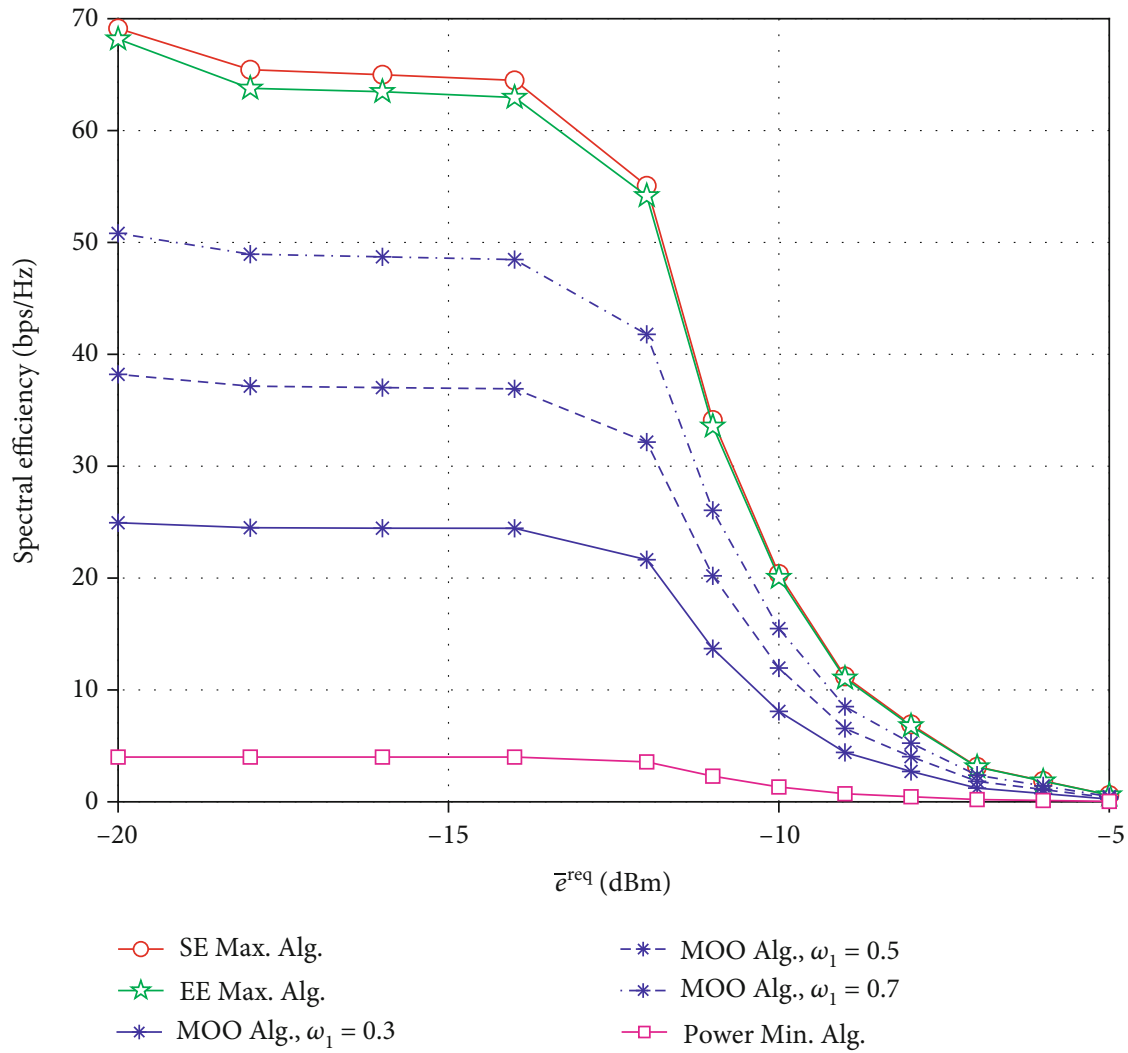

Figure 6: The data rate versus the minimum amount of harvested energy with $C^{\mathrm{FH}}=5(\mathrm{bps} / \mathrm{Hz})$.

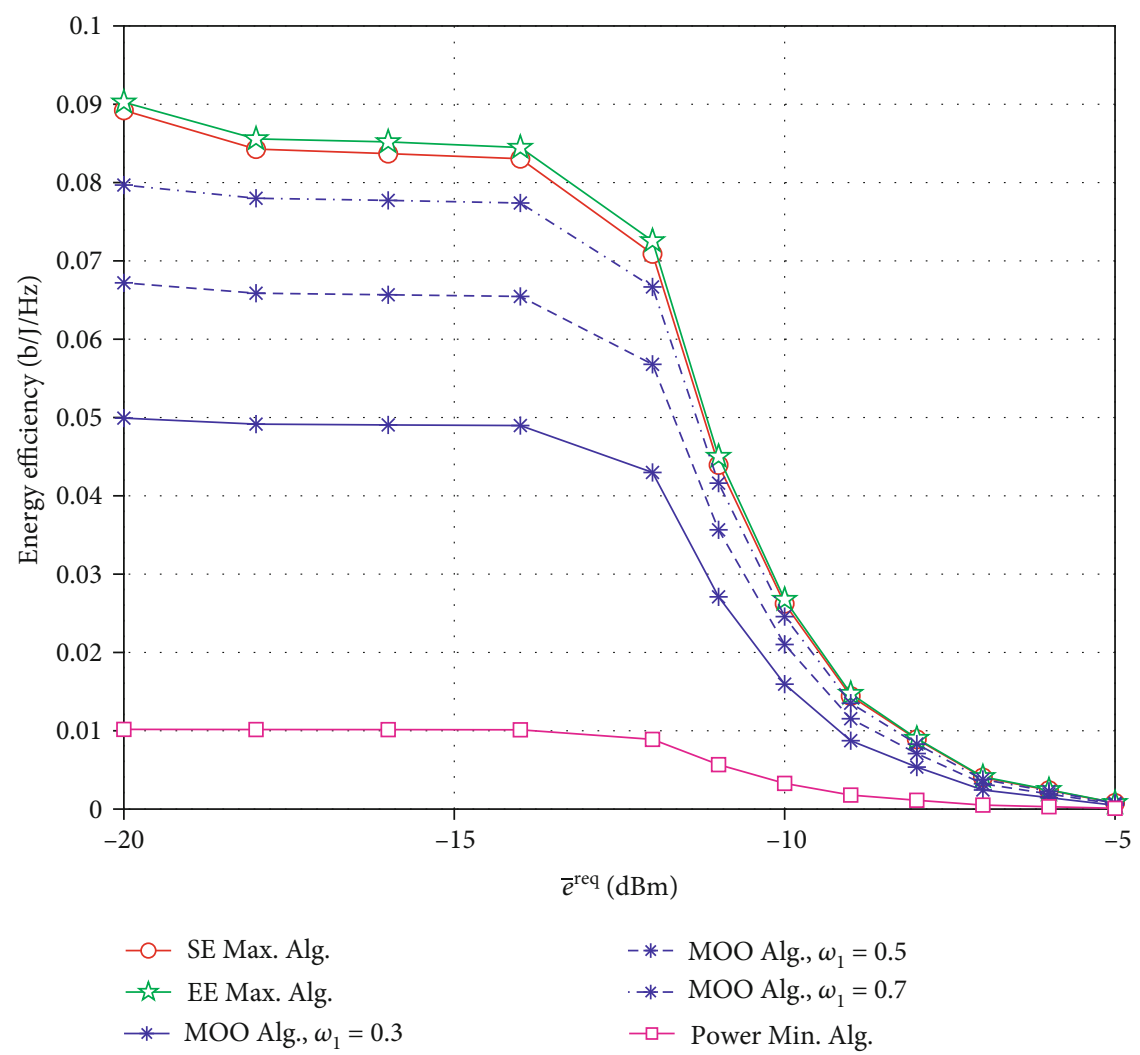

Figure 7: The energy efficiency versus the minimum amount of harvested energy with $C^{\mathrm{FH}}=5(\mathrm{bps} / \mathrm{Hz})$. 


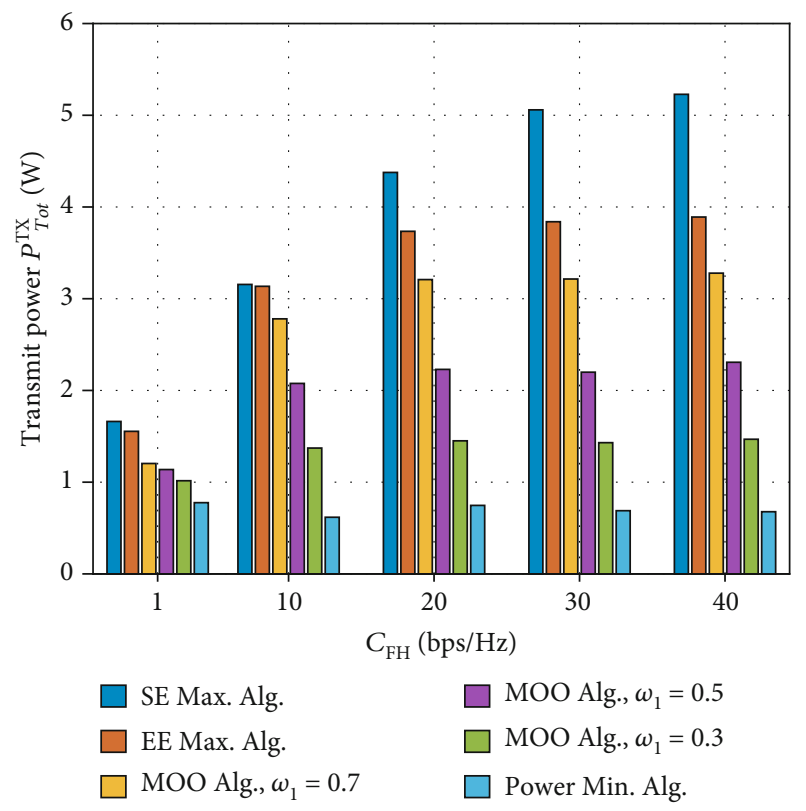

FIGURE 8: The average transmit power with various FH capacity levels and design methods.

cell and a pair of a DLU and a ULU is in each cell. Specifically, the DLU is randomly distributed within a circle with a radius of $r_{0}=10$ meters from RU while the ULU is randomized within its square cell area. In addition, the minimum distance between RU and any user is set as 5 meters. This cell setup implies that 4 DLUs, 4 ULUs, and 4 RUs are simulated. Furthermore, the transmit and receive antennas at each user is set as $N_{\ell_{U}}^{T x}=N_{k_{D}}^{R x}=2$ antennas for all $\ell_{U}$ and $k_{D}$ while each RU possesses $N_{\lambda_{R}}^{T x}=N_{\lambda_{R}}^{R x}=2$ antennas for all $\lambda_{R} \in \mathscr{R}$. The numbers of data streams are $d_{\ell_{U}}=N_{\ell_{U}}^{T x}$ and $d_{k_{D}}=N_{k_{D}}^{R x}$. For the sake of convenience, an identical power budget is set for all ULUs and all RUs, i.e., $P_{\ell_{U}, \max }=P_{\mathrm{U}, \max }, \forall \ell_{U} \in \mathcal{U}$ and $P_{\lambda_{R} \text {, max }}=$ $P_{R, \text { max }}, \forall \lambda_{R} \in \mathscr{R}$, respectively. In addition, the power budgets are set as $P_{R, \text { max }}=26 \mathrm{dBm}$ and $P_{\mathrm{U}, \text { max }}=23 \mathrm{dBm}[24,25]$. The power amplifier factors are $\xi_{\lambda_{R}}^{-1}=\xi_{\ell_{U}}^{-1}=2.8$ and $P_{\lambda_{R}}^{C i r}=84(\mathrm{~W})$ and $P_{\ell_{U}}^{C i r}=N_{\ell_{U}}^{T x} P_{\ell_{U}}^{C i r, D y n}+0.7 P_{\ell_{U}}^{C i r, D y n}$ with $P_{\ell_{U}}^{C i r, D y n}=8 \mathrm{dBW}$ for all $\ell_{U}, \lambda_{R}$ [31]. The noise floor is set as $\sigma_{k_{D}, a}^{2}=\sigma_{k_{D}, c}^{2}=\sigma_{R}^{2}=-$ $90 \mathrm{dBW}$ [51]. Regarding energy harvesters, the nonlinear $\mathrm{EH}$ parameters are set $\bar{P}_{k_{D}}^{D C}=24(\mathrm{~mW})$ and $\left(a_{k_{D}}, b_{k_{D}}\right)=($ $150,0.014)$ for all $k_{D} \in \mathscr{D}$ [57]. The required harvested energy is $e_{k_{D}}^{\text {req }}=\bar{e}^{\text {req }}$ for all $k_{D}$ and we also set $e^{\text {req }}=-20(\mathrm{dBm})$. In addition, we also set the required data rate of DL and UL channels as $\bar{r}_{k_{D}}^{\text {req }}=\bar{r}^{\text {req }}$ for all $\forall k_{D} \in \mathscr{D}$ and $\bar{r}_{R}=J \times \bar{r}^{\text {req. }}$. The maximum capable FH capacities and FH power scale factor are set as $C_{D, \lambda_{R}}=C_{U, \lambda_{R}}=C_{\mathrm{FH}}$ and $\alpha_{U, \lambda_{R}}^{\mathrm{FH}}=\alpha_{D, \lambda_{R}}^{\mathrm{FH}}=\alpha_{R}^{\mathrm{FH}}$, respectively, for all $\forall \lambda_{R} \in \mathscr{R}$. The required minimum rate is set to $\bar{r}^{\text {req }}=0.5(\mathrm{bps} / \mathrm{Hz})$. Without other statements, we also set $P_{U, \lambda_{R}, \max }^{\mathrm{FH}}=P_{D, \lambda_{R}, \max }^{\mathrm{FH}}=50(\mathrm{~W})$ and $C_{U, \lambda_{R}}=C_{D, \lambda_{R}}=100(\mathrm{Mbps})$ with $\lambda_{R} \in \mathscr{R}$ over the channel bandwidth of $10(\mathrm{MHz})$, which implies $\alpha_{R}^{\mathrm{FH}}=5(\mathrm{~W} /(\mathrm{bps} / \mathrm{Hz}))$ [30-32].
The channel matrix from node $A$ to node $B$ with distance $d_{A, B}$ meters are simulated as $\mathbf{M}_{A, B}=d_{A, B}^{-a / 2} \tilde{\mathbf{M}}_{A, B}$ with $\alpha$ being the path loss exponent. Meanwhile, the SI channel matrix at RU is conducted as $\mathbf{G}_{\lambda_{R}, \lambda_{R}}=10^{-C_{S I} / 20} \tilde{\mathbf{G}}_{\lambda_{R}, \lambda_{R}}$ where $C_{S I}$ (in decibel) denotes the SI suppression level. We also set $\alpha=4$ and $C_{S I}=90 \mathrm{~dB}$ [52]. The nominal small-scale fading matrix follows the Rician fading model as $\tilde{\mathbf{M}}=\sqrt{(1+K) / K \mathbf{M}^{\mathrm{LoS}}}+$ $\sqrt{1 /(1+K) \mathbf{M}^{N \text { LoS }}}$, where $K=10 \mathrm{~dB}$ is the Rician factor, the line-of-sight (LoS) term $\mathbf{M}^{\mathrm{LoS}}$ is a deterministic matrix, and each element of $\mathbf{M}^{N L o S}$ is an independent and identically distributed complex Gaussian variable with zero mean and unit variance, i.e., $\mathbf{M}^{N \operatorname{LoS}} \sim \mathscr{C} \mathscr{N}(0,1)$. In this paper, we utilize the far-field uniform linear antenna array model [51] $\mathbf{M}^{\mathrm{LoS}}=$ $\left[1, e^{j \theta_{r}}, e^{j 2 \theta_{r}}, \cdots, e^{j\left(N_{r}-1\right) \theta_{r}}\right]^{\dagger} \times\left[1, e^{j \theta_{t}}, e^{j 2 \theta_{t}}, \cdots, e^{j\left(N_{t}-1\right) \theta_{t}}\right]$, where $N_{r}$ and $N_{t}$ refer to the receiving and transmitting antennas, $\theta_{r}=2 \pi d \lambda^{-1} \sin \left(\phi_{r}\right)$ and $\theta_{t}=2 \pi d \lambda^{-1} \sin \left(\phi_{t}\right)$ with antenna spacing $d=0.5 \lambda$, carrier wavelength $\lambda$, the angle-of-arrival $\phi_{r}$, and the angle-of-departure $\phi_{t}$. The values of $\phi_{r}, \phi_{t}$ are randomly created in a range of $[0,2 \pi]$. Regarding the iteration algorithm, we set the error tolerance to be $\varepsilon=10^{-4}$ and the maximum iteration to be $\kappa_{\max }=150$. In the $\mathrm{MOO}$ algorithm, we set $\delta=0.005$.

4.2. System Performance Results. Firstly, the system SE and EE performances versus $\mathrm{FH}$ capacity with several design methods are illustrated in Figures 2 and 3, respectively. As can be seen in Figure 2, in the phenomenon of increasing FH capacity, the achievable sum rate first rapidly grows and then saturates for all design methods. From Figure 3, the system EE performance of the sum rate maximization algorithm is significantly degraded as the FH capacity is higher than a specific value, i.e., $12 \mathrm{bps} / \mathrm{Hz}$ for such system parameters. It is because that almost all of the quantity of the power budget is utilized to improve the system sum rate in the SE design (see also Figure 4), as well as the amount of consumed power is exponentially climbing with respect to the value of the $\mathrm{FH}$ rate as the rate-dependent $\mathrm{FH}$ power consumption model (see also Figure 5). Consequently, the improved gain of the data rate substantially drops compared to the steep acceleration in the amount of consumed power, and thus, system EE should be dramatically decreased. On the contrary, the EE optimization and EE-SE MOO approaches tend to stabilize system EE metric in the high regime of FH capacity even though a lower throughput should be obtained.

On the other hand, the average systems SE and EE as the function of the minimum required harvested energy are shown in Figures 6 and 7, respectively. It is noted that at the high value of required $\mathrm{EH} e^{\text {req, }}$, the problem might not be feasible. In this scenario, the systems SE and EE are set to equal zeros. It can be seen from these figures that both system performances degrade as the required $\mathrm{EH}$ grows. This is due to the fact that as the $\mathrm{EH}$ constraints are more restricted, the proportion signal for ID is reduced or/and more numbers of infeasibility of the related optimization problem can likely incur for random channel realizations. 


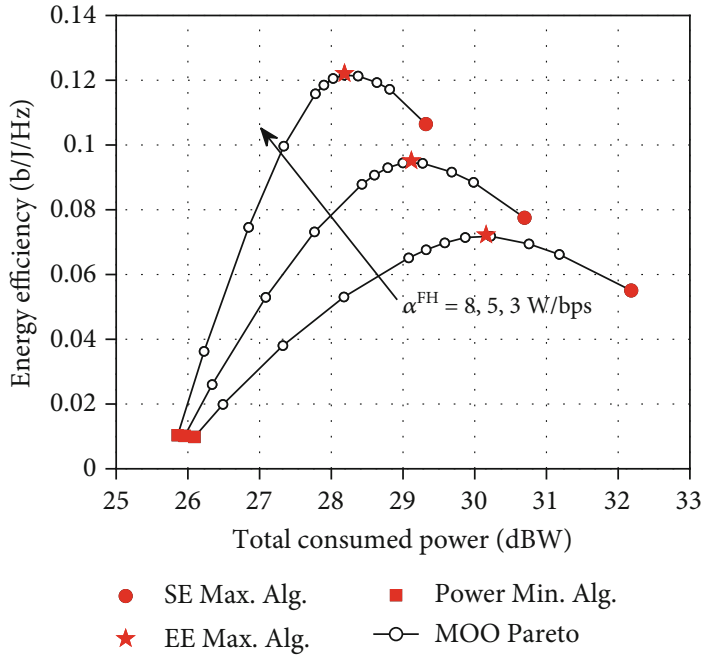

(a) EE and power consumption trade-off

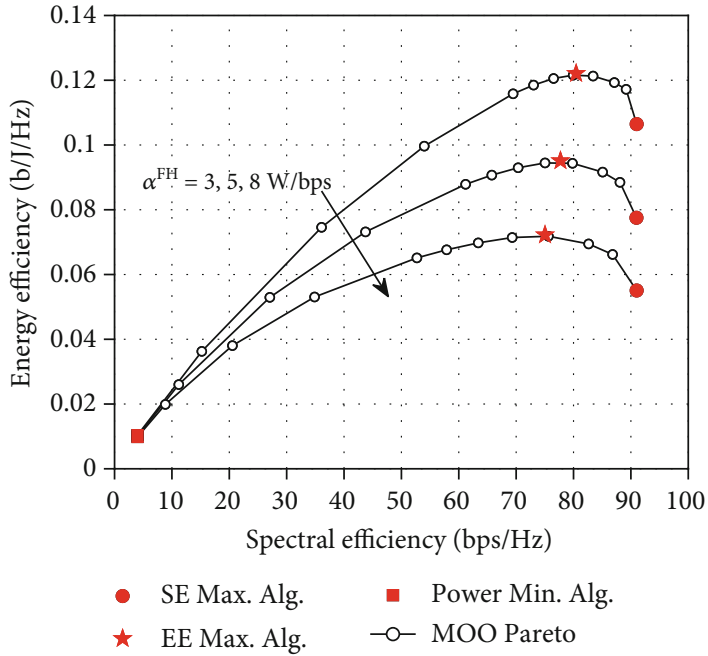

(b) EE and SE trade-off

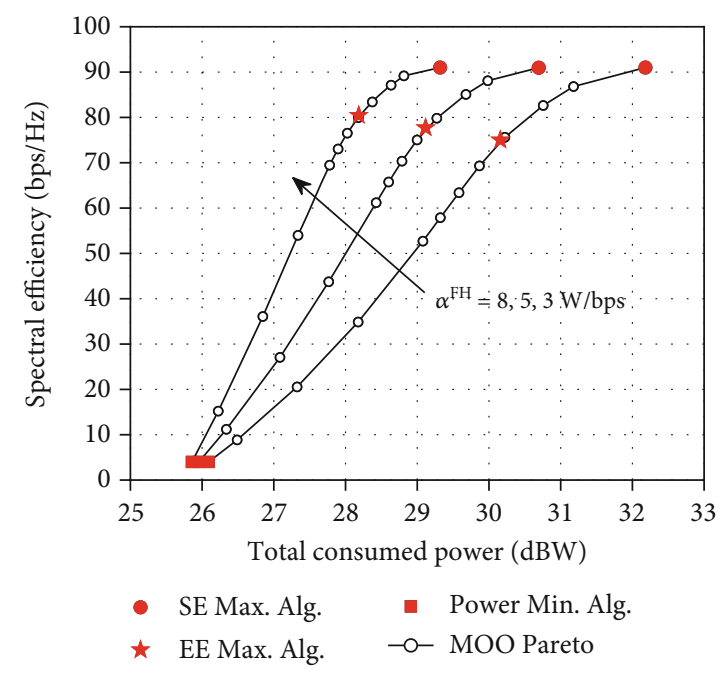

(c) SE and power consumption trade-off

Figure 9: The optimal Pareto sets with $\alpha^{\mathrm{FH}}=\{3,5,8\}$ (W/bps) and $C_{\mathrm{FH}}=20$ (bps/Hz).

4.3. Power Consumption Results. In order to obtain more insights into how algorithms worked on allocating power, various power consumption results are conducted and shown in this part. In Figure 4, the power consumption ratio is computed as the ratio of consumed power $\mathscr{P}_{\text {Tot }}$ to the power budget $\quad \mathscr{P}_{\text {Tot }}^{\text {budget }}=\sum_{\lambda_{R} \in \mathscr{R}} \xi_{\lambda_{R}}^{-1} P_{\lambda_{R}}+\sum_{\ell_{U} \in \mathcal{U}} \xi_{\ell_{U}}^{-1} P_{\ell_{U}}+\sum_{\lambda_{R} \in \mathscr{R}} \alpha_{U, \lambda_{R}}^{\mathrm{FH}}$ $C_{U, \lambda_{R}}+\alpha_{D, \lambda_{R}}^{\mathrm{FH}} C_{D, \lambda_{R}}$. From this figure, the power consumption ratio of SE optimization design approximately reaches at one for all FH configurations; this argument implies that the total power consumed by the SE maximization design and power budget is almost the same value. This can be concluded that the SE algorithm tends to completely utilize the overall available power to increase the system sum rate while the others reduce power consumption against ineffective power usage and to remain the system EE.

Figures 5 and 8 plot individual power expenditures consumed by the data transmission in (20) and the FH connection in (22), respectively, with different values of FH capacity. In general, there are upward trends in consuming power for both power consumption categories with the growing in FH capacity, and the separate design approaches have reached different power expenditures. It is because more data rate can be conveyed through the $\mathrm{FH}$ link possessing a higher capacity level and, thus, the maximum $\mathrm{FH}$ rate constraints are significantly slacker that results in more power being allocated. Accordingly, we can conclude from these two figures that the power consumption designed with the SE maximization algorithm reaches the highest value in all $\mathrm{FH}$ capacity setups whereas that of the power minimization design is the lowest one. Otherwise, the power figures corresponding either to the $\mathrm{EE}$ maximization or to EE-SE trade-off approaches constitute middle values between powers consumed by two former approaches since they are facilitated to achieve a balance between the rate and power utilities.

On the other hand, the power expending for FH links is remarkably greater than that for data transmission. For example, at $C_{F H}=10(\mathrm{bps} / \mathrm{Hz})$, the maximum power for data transmission only makes up at nearly $3(\mathrm{~W})$ while that for $\mathrm{FH}$ 


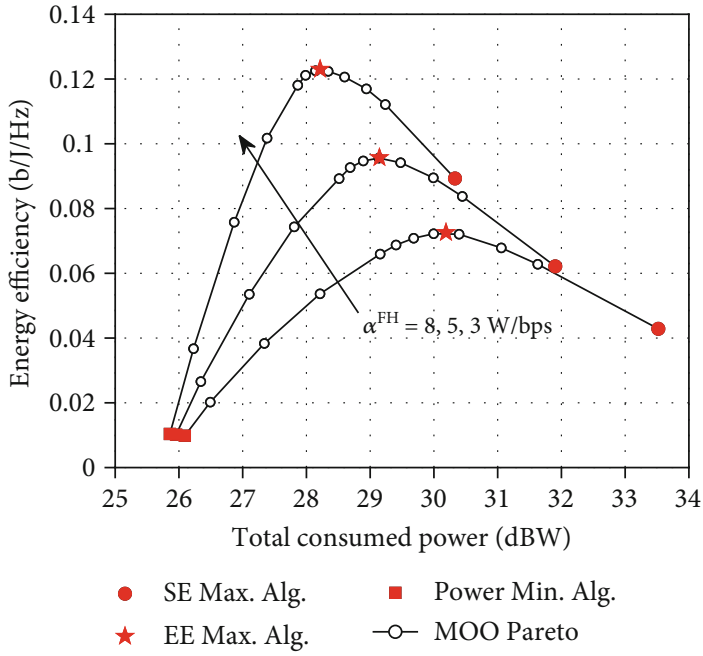

(a) EE and power consumption trade-off

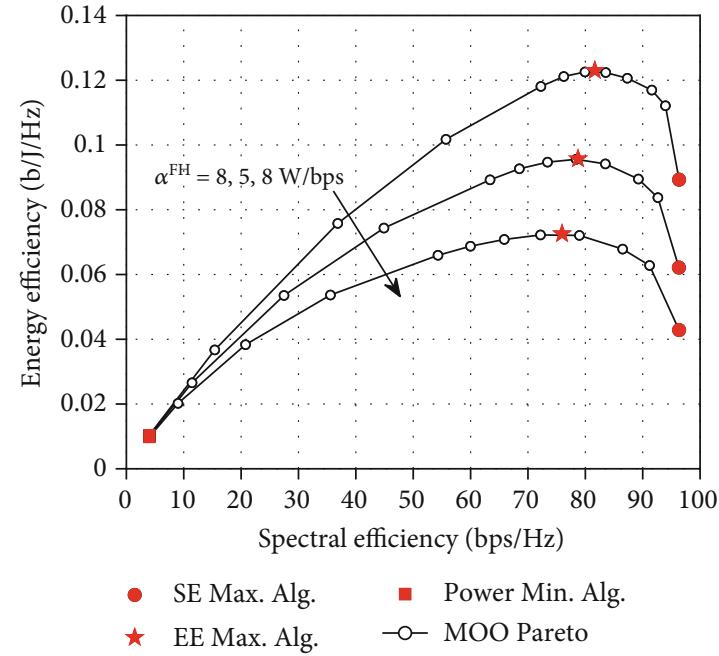

(b) EE and SE trade-off

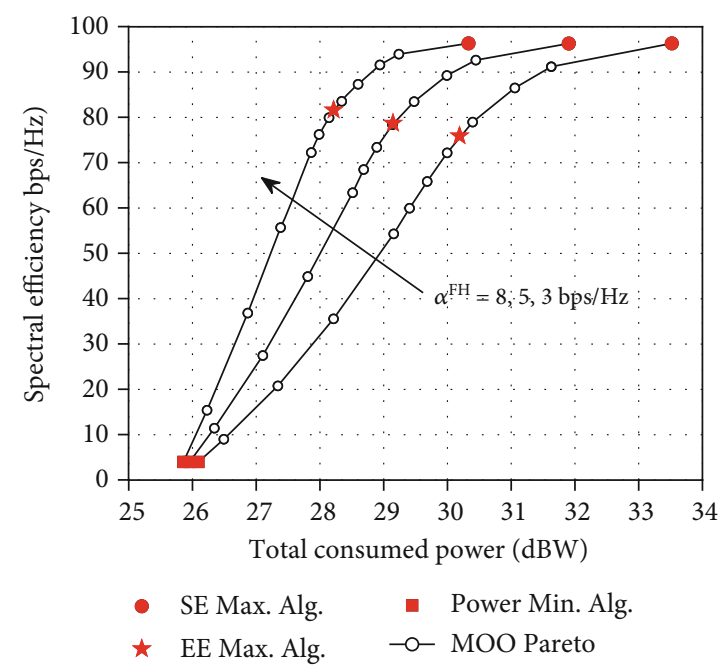

(c) SE and power consumption trade-off

Figure 10: The optimal Pareto sets with $\alpha^{\mathrm{FH}}=\{3,5,8\}(\mathrm{W} / \mathrm{bps})$ and $C_{\mathrm{FH}}=30(\mathrm{bps} / \mathrm{Hz})$.

links accounts for approximate 400 (W). These different levels are more significant at the high FH capacity. Therefore, it is necessary to take rate-dependent $\mathrm{FH}$ power consumption into consideration in investigating the CRAN systems.

4.4. Average Pareto for SE-EE Trade-Off Results. In this subsection, the optimal Pareto sets of various trade-offs between two among three considered metrics with several parameters are given. Specifically, the EE-power consumption, the energyspectral efficiency, and the spectrum-power consumption trade-offs with $C_{\mathrm{FH}}=20 \mathrm{bps} / \mathrm{Hz}$ or $C_{F \mathrm{H}}=30 \mathrm{bps} / \mathrm{Hz}$ are depicted in Figures 9 and 10, respectively. In these figures, the Pareto curves are obtained by changing the MOOweighted parameter, i.e., $0<\omega_{1}<1$, and the optimal solutions of SE, EE, and power consumption optimization problems are also depicted. As can be seen in the figures, as expected, a better Pareto region can be achieved either with a smaller value of $\alpha^{\mathrm{FH}}$ or with a larger $\mathrm{FH}$ capacity $C_{\mathrm{FH}}$. Furthermore, the energy-spectral efficiency and SE-power consumption Pareto sets are concave curves and have a maximum point; this opti- mum is almost the same as the optimal solution with the EE maximization design. Moreover, we can see in these figures that the SE maximization approach consumes the largest amount of power and reaches the greatest rate while the opposite is true for the power consumption minimization design method. Meanwhile, the EE maximization algorithm tends to obtain the maximum EE with an appropriate sum rate and affordable consumed power. Alternatively, the EE-SE MOO manner permits to adjust the priority in designing either with the SE maximization or with the power consumption minimization.

Finally, Figure 11 shows the EE-SE surface which is the trade-off between systems $\mathrm{EE}$ and $\mathrm{SE}$ with respect to various $\mathrm{FH}$ capacities $C_{\mathrm{FH}}$. As we can see, the more the capacity of the FH link, the larger EE-SE region we can obtain. This is because, in this case, the $\mathrm{FH}$ rate constraints are more relax and, thus, more power can be allocated. On the other hand, the figure also shows that there are three different optimum behaviors corresponding to SE maximization, EE maximization, and power consumption minimization design methods. 


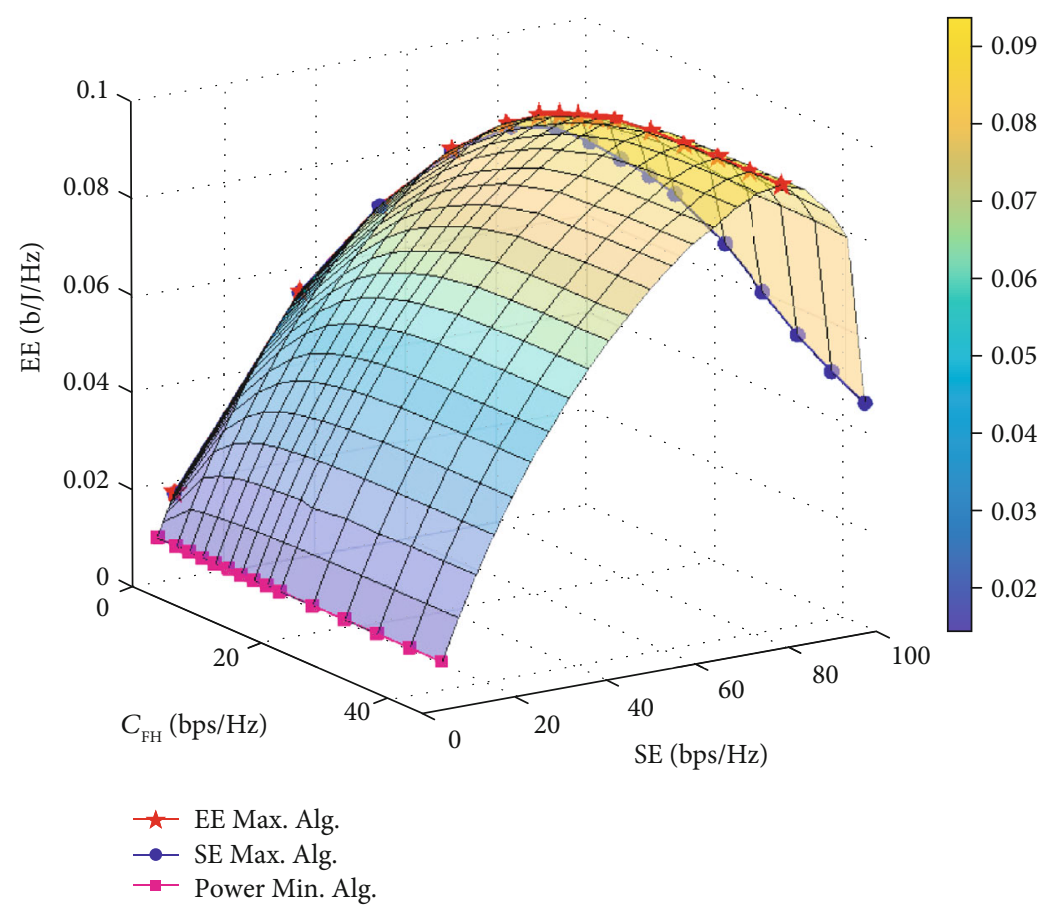

Figure 11: EE-SE trade-offs versus $C_{\mathrm{FH}}$.

Particularly, the system SE performance obtained by the SE maximization algorithm has a tendency to reach the highest sum rate even if its EE metric is considerably degraded in high FH capacity values. Alternatively, the EE approach tends to maximize the system EE with a suitable sum rate, whereas the power minimization method provides the lowest performance in terms of both SE and EE metrics for all $\mathrm{FH}$ capacity configurations since the only enough amount of power is used to achieve at the minimal system requirements. It is worth noting that the MOO algorithm provides an optimal Pareto curving surface in which the optimal solutions of the other approaches are considered as its particular points. Hence, the EE-SE trade-off-based MOO approach is actually a more general framework as compared to conventional SOO-based design methods which are sum rate maximization, EE maximization, and power consumption minimization approaches.

\section{Conclusion}

In this paper, we have considered a problem of EE-SE tradeoffs in FD MU-MIMO CRANs with SWIPT. The problem of interest is to design the transmit precoders, FH compression matrices, and $\mathrm{EH}$ power splitting factors to investigate the trade-offs of the system EE-SE. We have first formulated three common optimization problems by using SOO programming including the system sum rate maximization, power consumption minimization, and fractional sum rate to power consumption ratio maximization. Furthermore, we also have made use of the efficient MOO framework to simultaneously optimize both system sum-rate and overall power consumption. Each problem is constrained by the minimum QoS of the user data rate, transmit power budget, limited $\mathrm{FH}$ rate, and minimum harvested energy require- ment. All design problems are highly nonconvex because of the nonconcavity in user data rate expressions, nonconvex $\mathrm{FH}$ rate conditions, and nonconvex $\mathrm{EH}$ constraints and, thus, extremely intractable to tackle directly. For the nonconvex SOO problems, the inner convex quadratic approximations are conducted in the data rates, $\mathrm{FH}$ rates, and harvested energy to exploit the convexity in the optimization problems. Regarding the MOO EE-SE trade-off problem, it has first been recast to an SOO problem by applying the modified weighted Tchebycheff method, and then, the inner convex quadratic approximations are applied. As a result, SCQPbased iterative algorithms guaranteeing convergence are developed. The simulation results have revealed the design effectiveness in terms of the system EE in cases of sufficient large $\mathrm{FH}$ capacity as well as unveiled the interesting tradeoffs between the system energy and spectral efficiencies. Furthermore, the results have also illustrated that the conventional problems relying on SOO programming can be referred to as a special case of the considered MOO-based algorithm.

\section{Appendix}

\section{A. Concave Lower Bound of the System Rate}

Following ([52], Appendix A) and [53], with arbitrary $N \times N$ positive definite complex matrices $\mathbf{Y}$ and $\mathbf{Y}_{0}$ and $N \times M$ complex matrices $\mathbf{M}$ and $\mathbf{M}_{0}$, we obtain the global concave quadratic lower bound of a specific logarithm function below

$$
\begin{aligned}
\log _{2}\left|\mathbf{I}_{N}+\mathbf{M}^{\dagger} \mathbf{Y}^{-1} \mathbf{M}\right| & \geq a_{0}+\frac{2}{\ln (2)} \mathfrak{R}\left\{\operatorname{Tr}\left(\mathscr{A}_{0} \mathbf{M}\right)\right\} \\
& -\frac{1}{\ln (2)} \operatorname{Tr}\left(\mathscr{B}_{0} \mathbf{M} \mathbf{M}^{\dagger}+\mathscr{B}_{0} \mathbf{Y}\right),
\end{aligned}
$$


where $\mathscr{A}_{0}=\mathbf{M}_{0}^{\dagger} \mathbf{Y}_{0}^{-1}, \mathscr{B}_{0}=\mathbf{Y}_{0}^{-1}-\left(\mathbf{Y}_{0}+\mathbf{M}_{0} \mathbf{M}_{0}^{\dagger}\right)^{-1} \succeq 0$, and $a_{0}$ $\leq 0$ with

$$
a_{0}=\log _{2}\left|\mathbf{I}_{N}+\mathbf{M}_{0}^{\dagger} \mathbf{Y}_{0}^{-1} \mathbf{M}_{0}\right|-\frac{1}{\ln (2)} \operatorname{Tr}\left(\mathbf{M}_{0}^{\dagger} \mathbf{Y}_{0}^{-1} \mathbf{M}_{0}\right)
$$

Then, by applying (A.1) with substituting $\mathbf{H}_{k_{D}} \mathbf{F}_{k_{D}} \rightarrow \mathbf{M}, \boldsymbol{\Omega}_{k_{D}}$ $\rightarrow \mathbf{Y}, \mathbf{H}_{k_{D}} \mathbf{F}_{k_{D}}^{(\kappa)} \rightarrow \mathbf{M}_{0}$, and $\boldsymbol{\Omega}_{k_{D}}^{(\kappa)} \rightarrow \mathbf{Y}_{0}$, the DLU $k_{D}$ rate in (9) is thus lower bounded by a quadratic function in (33).

Regarding UL rate (18), upon defining $\mathbf{H}_{R, U} \triangleq\left[\mathbf{H}_{R, 1_{U}}\right.$, $\left.\mathbf{H}_{R, 2_{U}}, \cdots \mathbf{H}_{R, K_{U}}\right]$ and $\mathbf{F}_{U} \triangleq \operatorname{diag}\left\{\mathbf{F}_{\ell_{U}}, \ell_{U} \in \mathcal{U}\right\}$, this implies that

$$
\sum_{\ell_{U} \in \mathscr{U}} \mathbf{H}_{R, \ell_{U}} \mathbf{F}_{\ell_{U}} \mathbf{F}_{\ell_{U}}^{\dagger} \mathbf{H}_{R, \ell_{U}}^{\dagger}=\mathbf{H}_{R, U} \mathbf{F}_{U} \mathbf{F}_{U}^{\dagger} \mathbf{H}_{R, U}^{\dagger} .
$$

Then (18) can be rewritten, with $d_{U}=\sum_{\ell_{U} \in \mathcal{U}} d_{\ell_{U}}$, as

$$
R_{R}=\log _{2}\left|\mathbf{I}_{d_{U}}+\mathbf{F}_{U}^{\dagger} \mathbf{H}_{R, U}^{\dagger} \boldsymbol{\Omega}_{R}^{-1} \mathbf{H}_{R, U} \mathbf{F}_{U}\right|
$$

Next, by utilizing inequality (A.1) for (A.4), the UL rate (18) is globally lower bounded by the concave quadratic function in (35). This is end of proof.

\section{B. Convex Upper Bound of the FH Link Rate}

Firstly, equation (5) can be rewritten as the difference of two concavities

$$
g_{D, \lambda_{R}}=\log _{2}\left|\Theta_{\lambda_{R}}\right|-\log _{2}\left|\mathbf{Q}_{D, \lambda_{R}}\right|,
$$

where $\boldsymbol{\Theta}_{\lambda_{R}}=\sum_{k_{D} \in D} \mathbf{E}_{\lambda_{R}}^{\dagger} \mathbf{F}_{k_{D}} \mathbf{F}_{k_{D}}^{\dagger} \mathbf{E}_{\lambda_{R}}+\mathbf{Q}_{D, \lambda_{R}}$. Accordingly, its upper bound can be achieved by taking upper and lower bounds of first and second terms in the right-hand side (RHS) of (B.1), respectively. To this end, the upper bound of concave function $\log _{2}\left|\boldsymbol{\Theta}_{\lambda_{R}}\right|$ around $\left\{\mathbf{F}_{k_{D}}^{(\kappa)}, \mathbf{Q}_{D, \lambda_{R}}^{(\kappa)}: k_{D} \in \mathscr{D}\right\}$ is obtained by taking the first-order Taylor approximation as

$$
\log _{2}\left|\Theta_{\lambda_{R}}\right| \leq \log _{2}\left|\Theta_{\lambda_{R}}^{(\kappa)}\right|-\frac{N_{\lambda_{R}}^{T x}}{\ln (2)}+\frac{1}{\ln (2)} \operatorname{Tr}\left(\Theta_{\lambda_{R}}^{(\kappa),-1} \Theta_{\lambda_{R}}\right),
$$

where $\boldsymbol{\Theta}_{\lambda_{R}}^{(\kappa)}=\sum_{k_{D} \in \mathscr{D}} \mathbf{E}_{\lambda_{R}}^{\dagger} \mathbf{F}_{k_{D}}^{(\kappa)} \mathbf{F}_{k_{D}}^{(\kappa), \dagger} \mathbf{E}_{\lambda_{R}}+\mathbf{Q}_{D, \lambda_{R}}^{(\kappa)}$. In addition, the term $\log _{2}\left|\mathbf{Q}_{D, \lambda_{R}}\right|$ in (B.1) is lower bounded around $\mathbf{Q}_{D, \lambda_{R}}^{(\kappa)}$ as concave function ([58], (Eq. 40))

$\log _{2}\left|\mathbf{Q}_{D, \lambda_{R}}\right| \geq \log _{2}\left|\mathbf{Q}_{D, \lambda_{R}}^{(\kappa)}\right|+\frac{N_{\lambda_{R}}^{T x}}{\ln (2)}-\frac{1}{\ln (2)} \operatorname{Tr}\left(\mathbf{Q}_{D, \lambda_{R}}^{(\kappa)} \mathbf{Q}_{D, \lambda_{R}}^{-1}\right)$

Hence, by plugging these bounds into (B.1), we obtain the convex expression in (39) which is the upper bound of the $\mathrm{DL} \mathrm{FH}$ rate. On the other hand, with a similar procedure, the upper bound of UL FH rate is given in (41). This is end of proof.

\section{Linear Lower Bound of Harvested Energy at DLU}

Starting from (11), we first introduce auxiliary diagonal matrix variables $\mathbf{X}_{k_{D}} \geq \mathbf{I}_{N_{k_{D}}^{R x}}$ satisfying $\mathbf{I}_{N_{k_{D}}^{R x}}-\mathbf{W}_{k_{D}} \succeq \mathbf{X}_{k_{D}}^{-1} \succeq$ $\mathbf{0}_{N_{k_{D}}^{R x}}$ with $k_{D} \in \mathscr{D}$; the harvested energy of DLU $k_{D}$ is then lower projected by using the epigraph form as

$$
\begin{aligned}
E_{k_{D}} \geq & \sum_{i_{D} \in \mathscr{D}} \operatorname{Tr}\left(\left(\mathbf{H}_{k_{D}} \mathbf{F}_{i_{D}}\right)^{\dagger} \mathbf{X}_{k_{D}}^{-1} \mathbf{H}_{k_{D}} \mathbf{F}_{i_{D}}\right)+\sum_{\ell_{U} \in \mathcal{U}} \operatorname{Tr}\left(\left(\mathbf{G}_{k_{D}, \ell_{U}} \mathbf{F}_{\ell_{U}}\right)^{\dagger} \mathbf{X}_{k_{D}}^{-1} \mathbf{G}_{k_{D}, \ell_{U}} \mathbf{F}_{\ell_{U}}\right) \\
& -\operatorname{Tr}\left(\mathbf{W}_{k_{D}} \mathbf{H}_{k_{D}} \mathbf{Q}_{D} \mathbf{H}_{k_{D}}^{\dagger}\right)+\operatorname{Tr}\left(\mathbf{H}_{k_{D}} \mathbf{Q}_{D} \mathbf{H}_{k_{D}}^{\dagger}\right)-\sigma_{k_{D}, a}^{2} \operatorname{Tr}\left(\mathbf{W}_{k_{D}}\right)+N_{k_{D}}^{R x} \sigma_{k_{D}, a}^{2} .
\end{aligned}
$$

It is noted that the first three terms in the right-hand side of (C.1) are convex that makes it nonconcave. Therefore, in the following, we represent in detail the method to linearise these terms.

Firstly, by using the matrix inequality ([59], Appendix B and [58], Appendix A), for all $\mathbf{Y}_{0}, \mathbf{Y}, \mathbf{M}_{0}>0$ and $\mathbf{M}>0$, we get

$\mathbf{Y}^{\dagger} \mathbf{M}^{-1} \mathbf{Y} \pm \varphi(\mathbf{Y}, \mathbf{M}) \triangleq \mathbf{Y}_{0}^{\dagger} \mathbf{M}_{0}^{-1} \mathbf{Y}+\mathbf{Y}^{\dagger} \mathbf{M}_{0}^{-1} \mathbf{Y}_{0}-\mathbf{Y}_{0}^{\dagger} \mathbf{M}_{0}^{-1} \mathbf{M} \mathbf{M}_{0}^{-1} \mathbf{Y}_{0}$

over the trust region $\varphi(\mathbf{Y}, \mathbf{M}) \geq 0$. Then, by replacing $\mathbf{H}_{k_{D}}$ $\mathbf{F}_{i_{D}} \rightarrow \mathbf{Y}, \mathbf{X}_{k_{D}} \rightarrow \mathbf{M}, \mathbf{H}_{k_{D}} \mathbf{F}_{i_{D}}^{(\kappa)} \rightarrow \mathbf{Y}_{0}$, and $\mathbf{X}_{k_{D}}^{(\kappa)} \rightarrow \mathbf{M}_{0}$, we obtain

$$
\left(\mathbf{H}_{k_{D}} \mathbf{F}_{i_{D}}\right)^{\dagger} \mathbf{X}_{k_{D}}^{-1} \mathbf{H}_{k_{D}} \mathbf{F}_{i_{D}} \geq \varphi\left(\mathbf{H}_{k_{D}} \mathbf{F}_{i_{D}}, \mathbf{X}_{k_{D}}\right),
$$

with $\varphi\left(\mathbf{H}_{k_{D}} \mathbf{F}_{i_{D}}, \mathbf{X}_{k_{D}}\right)$ being already defined in (46). That yields

$$
\operatorname{Tr}\left(\left(\mathbf{H}_{k_{D}} \mathbf{F}_{i_{D}}\right)^{\dagger} \mathbf{X}_{k_{D}}^{-1} \mathbf{H}_{k_{D}} \mathbf{F}_{i_{D}}\right) \geq \operatorname{Tr}\left(\varphi\left(\mathbf{H}_{k_{D}} \mathbf{F}_{i_{D}}, \mathbf{X}_{k_{D}}\right)\right)
$$

Similarly, with the same steps, the second trace part in (C.1) can be lower bounded as

$$
\operatorname{Tr}\left(\left(\mathbf{G}_{k_{D}, \ell_{U}} \mathbf{F}_{\ell_{U}}\right)^{\dagger} \mathbf{X}_{k_{D}}^{-1} \mathbf{G}_{k_{D}, \ell_{U}} \mathbf{F}_{\ell_{U}}\right) \geq \operatorname{Tr}\left(\varphi\left(\mathbf{G}_{k_{D}, \ell_{U}} \mathbf{F}_{\ell_{U}}, \mathbf{X}_{k_{D}}\right)\right),
$$

with $\varphi\left(\mathbf{G}_{k_{D}, \ell_{U}} \mathbf{F}_{\ell_{U}}, \mathbf{X}_{k_{D}}\right)$ being given in (47).

Turning our attention into the third nonconcave nonconvex trace component in (C.1), by [60], (Eq. B.3), for all $\mathbf{Y}_{0}>0, \mathbf{Y}>0, \mathbf{M}_{0}>0$, and $\mathbf{M}>0$, we get

$$
\operatorname{Tr}\left(\mathbf{Y}^{\dagger} \mathbf{M}\right) \leq \frac{1}{2}\left(\left\|\mathbf{Y}_{0}^{-0.5} \mathbf{Y} \mathbf{M}_{0}^{0.5}\right\|^{2}+\left\|\mathbf{Y}_{0}^{0.5} \mathbf{M} \mathbf{M}_{0}^{-0.5}\right\|^{2}\right)
$$

By substituting $\mathbf{W}_{k_{D}} \rightarrow \mathbf{Y}, \mathbf{H}_{k_{D}} \mathbf{Q}_{D} \mathbf{H}_{k_{D}}^{\dagger} \rightarrow \mathbf{M}, \mathbf{W}_{k_{D}}^{(\kappa)} \rightarrow \mathbf{Y}_{0}$, and $\mathbf{H}_{k_{D}} \mathbf{Q}_{D}^{(\kappa)} \mathbf{H}_{k_{D}}^{\dagger} \rightarrow \mathbf{M}_{0}$, this trace term is then upper approximated, i.e., with $\vartheta\left(\mathbf{W}_{k_{D}}, \mathbf{Q}_{D}\right)$ being given in (48), by 


$$
\operatorname{Tr}\left(\mathbf{W}_{k_{D}} \mathbf{H}_{k_{D}} \mathbf{Q}_{D} \mathbf{H}_{k_{D}}^{\dagger}\right) \leq \vartheta\left(\mathbf{W}_{k_{D}}, \mathbf{H}_{k_{D}} \mathbf{Q}_{D} \mathbf{H}_{k_{D}}^{\dagger}\right)
$$

Finally, by plugging the bounded expressions in (C.4), (C.5), and (C.7) into (C.1), the linear lower bound formula for harvested energy is completed in (45). This is end of proof.

\section{Data Availability}

The data used to support the findings of this study are included within the article.

\section{Conflicts of Interest}

The authors declare that they have no conflicts of interest.

\section{Acknowledgments}

This research is funded by Ho Chi Minh City University of Technology-VNU-HCM under grant number T-ĐĐT2020-28. We would like to thank Ho Chi Minh City University of Technology (HCMUT), VNU-HCM, for the support of time and facilities for this study.

\section{References}

[1] M. Peng, X. Xie, Q. Hu, J. Zhang, and H. V. Poor, "Contractbased interference coordination in heterogeneous cloud radio access networks," IEEE Journal on Selected Areas in Communications, vol. 33, no. 6, pp. 1140-1153, 2015.

[2] M. Peng, Y. Sun, X. Li, Z. Mao, and C. Wang, "Recent advances in cloud radio access networks: system architectures, key techniques, and open issues," IEEE Communications Surveys \& Tutorials, vol. 18, no. 3, pp. 2282-2308, 2016.

[3] I. Al-Samman, R. Almesaeed, A. Doufexi, and M. Beach, "Heterogeneous cloud radio access networks: enhanced time allocation for interference mitigation," Wireless Communications and Mobile Computing, vol. 2018, Article ID 2084571, 17 pages, 2018.

[4] E. Björnson and E. Jorswieck, Optimal Resource Allocation in Coordinated Multi-Cell Systems, Now Publishers Inc, 2013.

[5] W. Shin, M. Vaezi, B. Lee, D. J. Love, J. Lee, and H. V. Poor, "Coordinated beamforming for multi-cell MIMO-NOMA," IEEE Communications Letters, vol. 21, no. 1, pp. 84-87, 2017.

[6] J. Zhao, T. Q. S. Quek, and Z. Lei, "Coordinated multipoint transmission with limited backhaul data transfer," IEEE Transactions on Wireless Communications, vol. 12, no. 6, pp. 27622775, 2013.

[7] D. Lopez-Perez, I. Guvenc, G. de la Roche, M. Kountouris, T. Q. S. Quek, and J. Zhang, "Enhanced intercell interference coordination challenges in heterogeneous networks," IEEE Wireless Communications, vol. 18, no. 3, pp. 22-30, 2011.

[8] Y. Shi, J. Zhang, and K. B. Letaief, "Group sparse beamforming for green Cloud-RAN," IEEE Transactions on Wireless Communications, vol. 13, no. 5, pp. 2809-2823, 2014.

[9] L. Liu and R. Zhang, "Optimized uplink transmission in multiantenna C-RAN with spatial compression and forward," IEEE Transactions on Signal Processing, vol. 63, no. 19, pp. 50835095, 2015.
[10] S. Lee, S. Park, and I. Lee, "NOMA systems with contentcentric multicast transmission for C-RAN," IEEE Wireless Communications Letters, vol. 7, no. 5, pp. 828-831, 2018.

[11] J. Kim, H. Lee, S. Park, and I. Lee, "Minimum rate maximization for wireless powered cloud radio access networks," IEEE Transactions on Vehicular Technology, vol. 68, no. 1, pp. 1045-1049, 2019.

[12] J. Kim, S. Park, O. Simeone, I. Lee, and S. Shamai Shitz, "Joint design of fronthauling and hybrid beamforming for downlink C-RAN systems," IEEE Transactions on Communications, vol. 67, no. 6, pp. 4423-4434, 2019.

[13] D. Yu, J. Kim, and S. Park, "An efficient rate-splitting multiple access scheme for the downlink of C-RAN systems," IEEE Wireless Communications Letters, vol. 8, no. 6, pp. 15551558, 2019.

[14] Y. Zhou and W. Yu, "Fronthaul compression and transmit beamforming optimization for multi-antenna uplink CRAN," IEEE Transactions on Signal Processing, vol. 64, no. 16, pp. 4138-4151, 2016.

[15] S. Park, O. Simeone, O. Sahin, and S. Shamai, "Joint decompression and decoding for cloud radio access networks," IEEE Signal Processing Letters, vol. 20, no. 5, pp. 503-506, 2013.

[16] S. Park, O. Simeone, O. Sahin, and S. Shamai, "Joint precoding and multivariate backhaul compression for the downlink of cloud radio access networks," IEEE Transactions on Signal Processing, vol. 61, no. 22, pp. 5646-5658, 2013.

[17] S. Park, O. Simeone, O. Sahin, and S. Shamai, "Inter-cluster design of precoding and fronthaul compression for cloud radio access networks," IEEE Wireless Communications Letters, vol. 3, no. 4, pp. 369-372, 2014.

[18] Z. Zhang, K. Long, A. V. Vasilakos, and L. Hanzo, "Full-duplex wireless communications: challenges, solutions, and future research directions," Proceedings of the IEEE, vol. 104, no. 7, pp. 1369-1409, 2016.

[19] R. Li, Y. Chen, G. Y. Li, and G. Liu, "Full-duplex cellular networks," IEEE Communications Magazine, vol. 55, no. 4, pp. 184-191, 2017.

[20] O. Simeone, E. Erkip, and S. Shamai, "Full-duplex cloud radio access networks: an information-theoretic viewpoint," IEEE Wireless Communications Letters, vol. 3, no. 4, pp. 413-416, 2014.

[21] W. Tang and S. Feng, "User selection and power minimization in full-duplex cloud radio access networks," IEEE Transactions on Signal Processing, vol. 67, no. 9, pp. 2426-2438, 2019.

[22] M. Mohammadi, H. A. Suraweera, and C. Tellambura, "Uplink/downlink rate analysis and impact of power allocation for full-duplex Cloud-RANs," IEEE Transactions on Wireless Communications, vol. 17, no. 9, pp. 5774-5788, 2018.

[23] C. Fang, P. Li, and K. Feng, "Joint interference cancellation and resource allocation for full-duplex cloud radio access networks," IEEE Transactions on Wireless Communications, vol. 18, no. 6, pp. 3019-3033, 2019.

[24] Y. Jeon, S. Park, C. Song, J. Moon, S. Maeng, and I. Lee, "Joint designs of fronthaul compression and precoding for fullduplex cloud radio access networks," IEEE Wireless Communications Letters, vol. 5, no. 6, pp. 632-635, 2016.

[25] A. C. Cirik, O. Taghizadeh, L. Lampe, and R. Mathar, "Fronthaul compression and precoding design for full-duplex cloud radio access network," IEEE Systems Journal, vol. 13, no. 2, pp. 1113-1124, 2019. 
[26] M. Zhao, Q. Shi, Y. Cai, and M. Zhao, "Joint transceiver design for full-duplex cloud radio access networks with SWIPT," IEEE Transactions on Wireless Communications, vol. 16, no. 9, pp. 5644-5658, 2017.

[27] Z. Chen, Z. Chen, L. X. Cai, and Y. Cheng, "Optimal beamforming design for simultaneous wireless information and power transfer in sustainable Cloud-RAN," IEEE Transactions on Green Communications and Networking, vol. 2, no. 1, pp. 163-174, 2018.

[28] K. Xu, Z. Shen, Y. Wang, X. Xia, and D. Zhang, "Hybrid timeswitching and power splitting SWIPT for full-duplex massive MIMO systems: a beam-domain approach," IEEE Transactions on Vehicular Technology, vol. 67, no. 8, pp. 7257-7274, 2018.

[29] S. Buzzi, C. I, T. E. Klein, H. V. Poor, C. Yang, and A. Zappone, "A survey of energy-efficient techniques for $5 \mathrm{G}$ networks and challenges ahead," IEEE Journal on Selected Areas in Communications, vol. 34, no. 4, pp. 697-709, 2016.

[30] B. Dai and W. Yu, "Energy efficiency of downlink transmission strategies for cloud radio access networks," IEEE Journal on Selected Areas in Communications, vol. 34, no. 4, pp. 10371050, 2016.

[31] T. T. Vu, D. T. Ngo, M. N. Dao, S. Durrani, D. H. N. Nguyen, and R. H. Middleton, "Energy efficiency maximization for downlink cloud radio access networks with data sharing and data compression," IEEE Transactions on Wireless Communications, vol. 17, no. 8, pp. 4955-4970, 2018.

[32] T. T. Vu, D. T. Ngo, M. N. Dao, S. Durrani, and R. H. Middleton, "Spectral and energy efficiency maximization for contentcentric C-RANs with edge caching," IEEE Transactions on Communications, vol. 66, no. 12, pp. 6628-6642, 2018.

[33] K. Nguyen, Q. Vu, M. Juntti, and L. Tran, "Energy efficiency maximization for C-RANs: discrete monotonic optimization, penalty, and $\ell_{0}$-approximation methods," IEEE Transactions on Signal Processing, vol. 66, no. 17, pp. 4435-4449, 2018.

[34] P. Luong, F. Gagnon, C. Despins, and L. Tran, "Joint virtual computing and radio resource allocation in limited fronthaul green C-RANs," IEEE Transactions on Wireless Communications, vol. 17, no. 4, pp. 2602-2617, 2018.

[35] P. Luong, F. Gagnon, C. Despins, and L. Tran, "Optimal energy-efficient beamforming designs for Cloud-RANs with rate-dependent fronthaul power," IEEE Transactions on Communications, vol. 67, no. 7, pp. 5099-5113, 2019.

[36] T. T. Vu, D. T. Ngo, M. N. Dao et al., "Energy-efficient fullduplex enabled cloud radio access networks," IEICE Transactions on Communications, vol. E103.B, no. 1, pp. 71-78, 2020.

[37] R. Marler and J. Arora, "Survey of multi-objective optimization methods for engineering," Structural and Multidisciplinary Optimization, vol. 26, no. 6, pp. 369-395, 2004.

[38] E. Bjornson, E. A. Jorswieck, M. Debbah, and B. Ottersten, "Multiobjective signal processing optimization: the way to balance conflicting metrics in 5G systems," IEEE Signal Processing Magazine, vol. 31, no. 6, pp. 14-23, 2014.

[39] X.-X. Nguyen, H. H. Kha, P. Q. Thai, and H. Q. Ta, "Multiobjective optimization for information-energy transfer tradeoffs in full-duplex multi-user MIMO cognitive networks," Telecommunication Systems, vol. 76, no. 1, pp. 85-96, 2021.

[40] T. N. Ha and H. H. Kha, "Harvested energy and spectral efficiency trade-offs in multicell MIMO wireless networks," Radioengineering, vol. 28, no. 1, pp. 331-339, 2019.
[41] J. Rubio, A. Pascual-Iserte, D. P. Palomar, and A. Goldsmith, "Joint optimization of power and data transfer in multiuser MIMO systems," IEEE Transactions on Signal Processing, vol. 65, no. 1, pp. 212-227, 2017.

[42] Y. Sun, D. W. K. Ng, J. Zhu, and R. Schober, "Multi-objective optimization for robust power efficient and secure fullduplex wireless communication systems," IEEE Transactions on Wireless Communications, vol. 15, no. 8, pp. 5511-5526, 2016.

[43] W. Wu, S. Wu, and B. Wang, "Robust multi-objective beamforming design for power efficient and secure communication in MU-MISO networks," IEEE Access, vol. 5, pp. 13277-13285, 2017.

[44] P. Luong, F. Gagnon, C. Despins, and L. Tran, "Optimal joint remote radio head selection and beamforming design for limited fronthaul C-RAN," IEEE Transactions on Signal Processing, vol. 65, no. 21, pp. 5605-5620, 2017.

[45] Y. Hao, Q. Ni, H. Li, and S. Hou, "Energy and spectral efficiency tradeoff with user association and power coordination in massive MIMO enabled HetNets," IEEE Communications Letters, vol. 20, no. 10, pp. 2091-2094, 2016.

[46] S. K. Goudos, P. D. Diamantoulakis, and G. K. Karagiannidis, "Multi-objective optimization in 5G wireless networks with massive MIMO," IEEE Communications Letters, vol. 22, no. 11, pp. 2346-2349, 2018.

[47] Z. Li, S. Gong, C. Xing, Z. Fei, and X. Yan, "Multi-objective optimization for distributed MIMO networks," IEEE Transactions on Communications, vol. 65, no. 10, pp. 4247-4259, 2017.

[48] D. W. K. Ng, E. S. Lo, and R. Schober, "Multiobjective resource allocation for secure communication in cognitive radio networks with wireless information and power transfer," IEEE Transactions on Vehicular Technology, vol. 65, no. 5, pp. 3166-3184, 2016.

[49] P. R. B. Gomes, A. L. F. de Almeida, J. P. C. da Costa, and R. T. de Sousa, "Joint DL and UL channel estimation for millimeter wave MIMO systems using tensor modeling," Wireless Communications and Mobile Computing, vol. 2019, Article ID 4858137, 13 pages, 2019.

[50] E. Boshkovska, D. W. K. Ng, N. Zlatanov, and R. Schober, "Practical non-linear energy harvesting model and resource allocation for SWIPT systems," IEEE Communications Letters, vol. 19, no. 12, pp. 2082-2085, 2015.

[51] H. H. M. Tam, H. D. Tuan, A. A. Nasir, T. Q. Duong, and H. V. Poor, "MIMO energy harvesting in full-duplex multi-user networks," IEEE Transactions on Wireless Communications, vol. 16, no. 5, pp. 3282-3297, 2017.

[52] H. H. M. Tam, H. D. Tuan, and D. T. Ngo, "Successive convex quadratic programming for quality-of-service management in full-duplex MU-MIMO multicell networks," IEEE Transactions on Communications, vol. 64, no. 6, pp. 2340-2353, 2016.

[53] H. D. Tuan, H. H. M. Tam, H. H. Nguyen, T. Q. Duong, and H. V. Poor, "Superposition signaling in broadcast interference networks," IEEE Transactions on Communications, vol. 65, no. 11, pp. 4646-4656, 2017.

[54] M. Grant, S. Boyd, and Y. Ye, Cvx: Matlab Software for Disciplined Convex Programming, 2008.

[55] W. Dinkelbach, "On nonlinear fractional programming," Management Science, vol. 13, no. 7, pp. 492-498, 1967.

[56] J. Xu and L. Qiu, "Energy efficiency optimization for MIMO broadcast channels," IEEE Transactions on Wireless Communications, vol. 12, no. 2, pp. 690-701, 2013. 
[57] J. Guo, H. Zhang, and X. Zhu, "Theoretical analysis of RF-DC conversion efficiency for class-f rectifiers," IEEE Transactions on Microwave Theory and Techniques, vol. 62, no. 4, pp. 977-985, 2014.

[58] V. Nguyen, H. D. Tuan, T. Q. Duong, H. V. Poor, and O. Shin, "Precoder design for signal superposition in MIMO-NOMA multicell networks," IEEE Journal on Selected Areas in Communications, vol. 35, no. 12, pp. 2681-2695, 2017.

[59] U. Rashid, H. D. Tuan, H. H. Kha, and H. H. Nguyen, "Joint optimization of source precoding and relay beamforming in wireless MIMO relay networks," IEEE Transactions on Communications, vol. 62, no. 2, pp. 488-499, 2014.

[60] H. Yu, H. D. Tuan, T. Q. Duong, T. Q. Duong, Y. Fang, and L. Hanzo, "Improper gaussian signaling for integrated data and energy networking," IEEE Transactions on Communications, vol. 68, no. 6, pp. 3922-3934, 2020. 